IFT-UAM/CSIC-08-74

UPR-1202-T

\title{
Stringy Instantons and Yukawa Couplings in MSSM-like Orientifold Models
}

\author{
L.E. Ibáñez ${ }^{1}$ and R. Richter ${ }^{2}$ \\ 1 Departamento de Fisica Teórica and Instituto de Fisica Teórica UAM-CSIC, \\ Universidad Autónoma de Madrid, Cantoblanco, 28049 Madrid, Spain \\ ${ }^{2}$ Department of Physics and Astronomy, University of Pennsylvania, \\ Philadelphia, PA 19104-6396, USA \\ ibanez@madriz1.ft.uam.es, rrichter@physics.upenn.edu
}

\begin{abstract}
Type IIA orientifold constructions with intersecting D6-branes and their IIB duals in terms of magnetized D9/D7-branes constitute one of the most promising avenues for the construction of semirealistic MSSMlike compactifications. One generic problem with these constructions is, that there are many Yukawa couplings, which vanish due to additional $U(1)$ symmetries in the theory. In this paper we consider a number of such settings and study, under what conditions stringy instanton effects can give rise to non-perturbative contributions to the Yukawa couplings, so that all perturbatively forbidden terms are induced. We find specific settings, in which indeed Yukawa couplings for all fermions are obtained. For some cases we provide specific local examples of rigid $O(1)$ instantons within the $T^{6} / \mathbb{Z}_{2} \times \mathbb{Z}_{2}^{\prime}$ toroidal orientifold with torsion, giving rise to the required amplitudes. A potential problem in these settings is, that the same instantons, providing for Yukawa coupling contributions, may give rise to too large $\mu$-terms for the Higgs multiplets. We show how this problem may be overcome in explicit models with a doubled Higgs system.
\end{abstract}




\section{Introduction}

Due to their very appealing geometrical interpretation intersecting brane worlds (for recent reviews on this subject see [1-3]) have been a popular playground for realistic model building. In such models the gauge groups appear on stacks of D6-branes, filling out the four-dimensional spacetime and wrapping three-cycles in the six-dimensional internal compactification manifold. Chiral matter arises at intersections of two stacks of D6-branes, wrapping different three-cycles in the internal manifold and their multiplicity is encoded in the intersection number of the respective three-cycles.

Over the last decade many intersecting brane models, giving rise to MSSMand GUT-like spectrum, have been constructed using mostly toroidal orbifolds as compactification manifold 1 . Given these, the next question is, if we can reproduce finer details of the MSSM, such as Yukawa couplings as well as their hierarchies. For intersecting D-brane constructions the Yukawa couplings for chiral matter fields can be extracted from string amplitudes [10-12]. These are suppressed by the open string world-sheet instantons connecting the three intersecting branes $[6,13]$. The latter potentially give rise to interesting Yukawa hierarchies, which, in principle, could reproduce the quark mass matrices observed in the MSSM.

However, for specific intersecting D-brane constructions on toroidal backgrounds, one often observes a factorization in terms of family indices of the Yukawa couplings. This is happens for instance if non-trivial intersections appear in different two tori of the orientifold [13]. The factorization of the Yukawa couplings for the different matter fields (u-quark, d-quark, electron and neutrino) allows only one of the three families to acquire dirac masses. While this might explain the hierarchy between the masses of the heaviest family compared to the two lightest family [13], one still faces the problem, how to generate masses for the other families. As demonstrated in [14] (see also [15-17]) additional Higgs pairs might overcome this issue and for appropriate values of the open and closed string moduli give rise to desired Yukawa hierarchies. Let us emphasize that for constructions on more general backgrounds such factorizations of Yukawa couplings is expected to be absent.

Nevertheless, in the existing constructions typically some of the Yukawa couplings are forbidden due to the violation of global $U(1)$ 's, ruling them out of being realistic. These global $U(1)$ 's are remnants of the Green-Schwarz mechanism, a necessary ingredient to ensure the cancellation of pure abelian as well as mixed anomalies.

Recently, it has been realized that D-brane instantons can break these global $U(1)$ 's and induce otherwise forbidden couplings [18-21]. These non-perturbative effects cannot be described as gauge instantons in field theory, thus are purely

\footnotetext{
${ }^{1}$ For original work on non-supersymmetric intersecting D-branes, see [4-7] and for chiral supersymmetric ones see $[8,9]$.
} 
stringy. For type IIA compactification the relevant class of instantons are, so called, E2-instantons, wrapping a three-cycle in the internal manifold and being localized in four-dimensional spacetime. Under a $U(1)_{a}$ gauge transformation the action of such an E2-instanton, wrapping the three-cycle $\pi_{E 2}$, transforms as

$$
e^{-S_{E 2}}=\exp \left[\frac{2 \pi}{\ell_{s}^{3}}\left(-\frac{1}{g_{s}} \operatorname{Vol}_{\pi_{E 2}}+i \int_{\pi_{E 2}} C^{(3)}\right)\right] \longrightarrow e^{i Q_{a}(E 2) \Lambda_{a}} e^{-S_{E 2}}
$$

with 2

$$
Q_{a}(E 2)=-N_{a} \pi_{E 2} \circ\left[\pi_{a}-\pi_{a}^{\prime}\right]
$$

Here $\pi_{a}$ and $\pi_{a^{\prime}}$ denote the three-cycles which the brane $a$ and its orientifold image $a^{\prime}$ wrap and $N_{a}$ is the number of $D 6$-branes for stack $a$. A superpotential term can be induced if the product

$$
W^{n p}=\prod_{i} \Phi_{a_{i} b_{i}} e^{-S_{E 2}}
$$

is invariant under all $\mathrm{U}(1)$ gauge transformations, thus if the $E 2$ instanton compensates for all global U(1)'s carried just by the product $\prod_{i} \Phi_{a_{i} b_{i}}$. Due to their non-perturbative nature these couplings are suppressed, which potentially gives an explanation for various hierarchies observed in nature.

Various applications of these novel non-perturbative effects in different branches of the string landscape have appeared [18-65] 3. These include the explicit generation of perturbatively forbidden couplings, such as Majorana masses for the right-handed Neutrinos, $\mu$-terms for the MSSM Higgs sector or Yukawa-couplings of type $\mathbf{1 0} \cdot \mathbf{1 0} \cdot \mathbf{5}_{H}$ in $S U(5)$-like GUT models. Furthermore, stringy instanton effects play a crucial role in the study of dynamical supersymmetry breaking, as well as moduli stabilization. Other, more formal aspects, involve lifting of additional zero modes via fluxes, multiinstanton contributions as well as global issues of these non-perturbative effects.

In this work, we analyze various D-brane constructions presented in [71] and [72], which give rise to the MSSM spectrum or extensions of it, with respect to their Yukawa couplings 4 . In case the desired couplings are perturbatively

\footnotetext{
${ }^{2}$ Note that, in contrast to [18], there is an additional minus sign in (2), which is due to the fact that a positive intersection number $I_{E 2 a}$ corresponds to the transformation behavior $(E 2, \bar{a})$ of the charged fermionic zero modes, rather than $(\overline{E 2}, a)$. In the sequel we denote the real part of $S_{E 2}$, the instanton suppression factor, $S_{E 2}^{c l}$.

${ }^{3}$ For two reviews on the subject of novel stringy instanton effects see [66,67]. For related earlier work see [68-70].

${ }^{4}$ In the alternative MSSM-like model of ref. $[13,73,74]$ there are no Yukawa couplings forbidden by any $U(1)$ symmetries and hence charged instantons cannot induce any extra Yukawa coupling.
} 
forbidden, since they violate global $U(1)$ selection rules, we present conditions under which these couplings can be generated via E2-instantons. We investigate the implications of these non-perturbative effects on the four-dimensional phenomenology and compare them to the observed experimental structure. Furthermore, we present local realizations on the $T^{6} / \mathbb{Z}_{2} \times \mathbb{Z}_{2}^{\prime}$ orbifold with discrete torsion [75], in which various couplings are generated by rigid $O(1)$ instantons. We demonstrate for these local models that the instanton indeed have the right suppression factor to yield to results compatible with experiments.

Let us point out that the derived results also apply to the T-dual Type I framework, where the superpotential receives contributions from $E 1$ and $E 5$ instantons. This corner of the string landscape is amenable to algebraic geometry techniques and has proven to be more promising for constructions of globally consistent semi-realistic string vacua, which exhibit stringy instanton corrections to the superpotential $[40,57]$. The $O(1)$ instantons in Type IIA correspond to $E 1$ instantons in Type I. Thus all the conditions on the E2-instantons presented in the course of this work can be easily translated into the Type I framework, where the Yukawa coupling inducing instanton is a rigid $E 1$ instanton.

\section{E2-instanton}

As pointed out in the introduction, E2-instantons with the right charge under the global $U(1)$ can generate a superpotential term of the form

$$
W^{n p}=\prod_{i} \Phi_{a_{i} b_{i}} e^{-S_{E 2}},
$$

which is otherwise forbidden. Note that the condition of carrying the right charge is just a necessary one, but not sufficient. Whether an instanton indeed contributes to the superpotential, depends crucially on its zero mode structure. After briefly recalling the zero mode structure for a generic instanton $[25,29]$, we review the computation of a non-perturbative superpotential contribution, induced by a rigid $O(1)$ instanton [18].

An E2 instanton, wrapping a generic three-cycle $\pi_{E 2}$, gives rise to the four bosonic zero modes $x^{\mu}$ due to the breakdown of four-dimensional Poincaré invariance. They are accompanied with four fermionic zero modes $\theta^{\alpha}$ and $\bar{\tau}^{\dot{\alpha}}$ indicating the breakdown of the $\mathcal{N}=2$ supersymmetry, preserved by the Calabi-Yau manifold, down to $\mathcal{N}=1$ supersymmetry. In addition the instanton $E 2$ exhibits $b_{1}\left(\pi_{E 2}\right)$ zero modes associated with deformations of the special Lagrangian cycle $\pi_{E 2}$. Moreover, in the presence of multiple instantons there appear zero modes at intersections of two instantons.

Finally, there are fermionic zero modes charged under the $D 6$ branes, which we call charged fermionic zero modes in the sequel. These arise at intersections between the instanton E2 and the D6-branes. Due to the GSO-projection only 
the chiral fermion is present, which is crucial for the holomorphicity of the superpotential. Adding up the D6-brane charge of all charged fermionic zero modes, gives the total charge of the instanton $Q_{a}(E 2)$, which coincides with (2).

The non-perturbative contribution is given by the path integral over all instanton zero modes, thus in order to give rise to F-terms we expect all uncharged zero modes, apart from $x^{\mu}$, and $\theta^{\alpha}$, to be projected out or lifted. There are various ways to ensure the absence of these additional undesired zero modes, such as lifting via fluxes $[35,56,58,62]$, via additional instantons [35, 42, 49, 51] or via additional interaction terms in case the instanton wraps a cycle which coincides with one of the spacetime filling D6-branes [23,43].

Here we focus on a class of instantons wrapping a rigid, orientifold invariant cycle in the internal manifold, so called rigid $O(1)$ instantons. For those the undesired $\bar{\tau}^{\dot{\alpha}}$ modes get projected out [21,27-29] and due to the rigidity of the three-cycle there are no zero modes associated with the deformation of the threecycle. Moreover, in that case the $U(1)_{a}$ charge of the instanton $E 2$ simplifies to

$$
Q_{a}(E 2)=-N_{a} \pi_{E 2} \circ \pi_{a}
$$

due to the identification of the $E 2-a$ and $E 2-a^{\prime}$ sector. Then the pathintegral takes the form

$$
W^{n p}=\int d^{4} x d^{2} \theta \prod_{i} d \lambda_{a_{i}} e^{-S_{E 2}^{c l}} e^{-S^{i n t}}
$$

Here $e^{-S_{E 2}^{c l}}$ is the suppression factor 5 and $S^{\text {int }}$ stands for all interaction terms involving the charged instanton zero modes $\lambda_{a_{i}}$ and matter fields $\Phi$. Due to holomorphicity of the superpotential, $S^{\text {int }}$ contains only disk amplitudes, carrying exactly two charged zero modes $\lambda$, and annulus contributions with no charged zero modes inserted [18]. The latter contribution has been calculated in $[22,23,30]$ and is related to the regularized threshold correction to the gauge coupling of a D6-brane wrapping the same cycle as the instanton. Thus the computation of the non-perturbative superpotential contribution results into the calculation of various disk diagrams involving the charged zero modes.

\section{Four-stack models}

A very natural way of realizing the MSSM is to embed the matter content at intersections of 4 stacks of $D 6$-branes giving rise to the gauge symmetry

$$
U(3)_{a} \times U(2)_{b} \times U(1)_{c} \times U(1)_{d} .
$$

\footnotetext{
${ }^{5}$ The suppression factor $e^{-S_{E 2}^{c l}}$ is the real part of $S_{E 2}$ in (4).
} 
The left-handed quarks $q_{L}$ are localized at the intersection of brane $a$ and $b$ or its orientifold image $b^{\prime}$, while the right-handed quarks, $u_{R}$ and $d_{R}$, arise at intersections of brane $a$ with one of the $U(1)$ branes and its orientifold image. The left-handed leptons are charged under the $U(2)$ and neutral under $U(3)$, thus appear at intersections between brane $b$ and one of the $U(1)$ branes. Finally, the right-handed electron $E_{R}$ and the right-handed neutrino $N_{R}$, both singlet under $U(3)$ and $U(2)$ arise at intersections of two $U(1)$ branes.

Tadpole cancellation, essential for global consistency, requires equal number of fields transforming as fundamental, $a$, and as anti-fundamental, $\bar{a}$, under the gauge group $U\left(N_{a}\right) \sqrt{6}$. This hold also true $U(2)$, whose fundamental representation is real, thus equal to the anti-fundamental one.

For a subclass of 4-stack models the constraint, mentioned above, can be satisfied within the MSSM matter content. This can be achieved by requiring that exactly 2 generations of the left-handed quarks $q_{L}$ arise from the sector $a b^{\prime}$, while the third family is localized at the intersection of brane $a$ and $b$. Ensuring that the number of fundamentals is equal the number of anti-fundamentals puts additional constraints on the transformation behavior of the matter and Higgs fields. Even though, the homology classes of the D-branes $a, b, c$ and $d$ alone might not cancel all the tadpoles, one may assume that one can find additional D-branes ensuring global consistency, in such a way, that they do not give rise to any additional chiral exotics charged under the matter D6-branes. For such local setups it is possible to investigate various phenomenological aspects without knowing the details of the global realization.

In this paper we discuss three different setups which give rise to the MSSM and extensions of it. We analyze which Yukawa couplings are perturbatively realized and investigate under what circumstances an instanton induces the perturbatively missing, but desired couplings. Let us list for all three setups the matter, charged under $S U(3)$ and $S U(2)$

$$
\begin{aligned}
\text { (1) } 1 \times Q_{l}=(3, \overline{2}) \quad 2 \times q_{l}=(3,2) \quad 3 \times l=(1, \overline{2}) \\
\\
1 \times\left(H_{d}+H_{u}\right)^{N=2}=(1, \overline{2})+(1,2) \\
\text { (2) } 1 \times Q_{l}=(3, \overline{2}) \quad 2 \times q_{l}=(3,2) \quad 2 \times l=(1,2) \quad 1 \times L=(1, \overline{2}) \\
1 \times H_{u}=(1, \overline{2}) \quad 1 \times H_{d}=(1, \overline{2}) . \\
\text { (3) } 1 \times Q_{l}=(3, \overline{2}) \quad 2 \times q_{l}=(3,2) \quad 2 \times l=(1,2) \quad 1 \times L=(1, \overline{2}) \\
2 \times H_{u}=(1, \overline{2}) \quad 2 \times H_{d}=(1, \overline{2}) .
\end{aligned}
$$

Subsequently, we refer to these three setups as the square quiver, triangle quiver and triangle quiver with doubled Higgs sector, respectively [71,72].

\footnotetext{
${ }^{6}$ Here we assume the absence of symmetrics and anti-symmetrics under $U\left(N_{a}\right)$
} 
Note, that the second setup, the triangle quiver, does not fulfill the constraint, equal number of fundamentals and anti-fundamentals for the $S U(2)$. Thus in this case additional exotics, charged under the MSSM gauge groups, are required to ensure global consistency. In the third setup we allow for an additional Higgs pair. Later we will see that the second Higgs pair is crucial to overcome a phenomenological problem encountered in the triangle quiver with just one Higgs pair.

All three setups are analyzed with respect to their Yukawa couplings. We will see, that various MSSM couplings are absent due to violation of global $U(1)$ selection rules. For these perturbatively forbidden couplings we discuss under what circumstances they can be generated non-perturbatively. Specifically, we present necessary conditions on the instanton zero mode structure and analyze their phenomenology with respect to the experimentally observed hierarchies.

For the triangle quivers, setups (2) and (3), we give local realizations. As background we choose the orientifold $T^{6} / \mathbb{Z}_{2} \times \mathbb{Z}_{2}^{\prime}$ with Hodge numbers $\left(h_{11}, h_{12}\right)=$ $(3,51)$, often called the $T^{6} / \mathbb{Z}_{2} \times \mathbb{Z}_{2}^{\prime}$ with discrete torsion, which gives rise to rigid cycles [75]. A brief introduction, which covers all necessary ingredients for the construction of the local realizations, is given in appendix A. We show that these local realizations exhibit instantons which carry the right zero mode structure to induce the perturbatively forbidden, but desired couplings.

\subsection{Square quiver}

Such a setup can be realized by the intersection numbers $[71,72]$

$$
\begin{gathered}
I_{a b}=1 \quad I_{a b^{\prime}}=2 \quad I_{a c}=-3 \quad I_{a c^{\prime}}=-3 \quad I_{b d}=0 \quad I_{b d^{\prime}}=-3 \\
I_{b c}=0 \quad I_{b c^{\prime}}=0 \quad I_{b c^{\prime}}^{\mathcal{N}=2}=1 \quad I_{c d}=-3 \quad I_{c d^{\prime}}=3 .
\end{gathered}
$$

Note that these intersection numbers correspond to a possible SUSY extension of the class of non-SUSY models constructed in [71]. They give rise to the spectrum displayed in table 1 .

Generically, the anomalous $U(1)$ 's acquire a mass via the Green-Schwarz mechanism and survive as global symmetries. To achieve the presence of the standard model gauge groups, we require that the combination

$$
U(1)_{Y}=\frac{1}{3} U(1)_{a}-U(1)_{c}+U(1)_{d}
$$

which corresponds to the hypercharge, remains massless. For simplicity we assume, that this is the only massless $U(1)$. Thus the gauge symmetry in four dimensional space-time is

$$
S U(3)_{a} \times S U(2)_{b} \times U(1)_{Y} .
$$

\footnotetext{
${ }^{7}$ Positive intersection number $I_{a b}=\pi_{a} \circ \pi_{b}$ corresponds to a chiral superfield transforming as $(a, \bar{b})$ under the gauge groups $U\left(N_{a}\right)$ and $U\left(N_{b}\right)$.
} 


\begin{tabular}{|c|c|c|c|}
\hline sector & matter fields & transformation & multiplicity \\
\hline \hline$a b$ & $Q_{L}$ & $(a, \bar{b})$ & 1 \\
\hline$a b^{\prime}$ & $q_{L}$ & $(a, b)$ & 2 \\
\hline$a c$ & $u_{R}$ & $(\bar{a}, c)$ & 3 \\
\hline$a c^{\prime}$ & $d_{R}$ & $(\bar{a}, \bar{c})$ & 3 \\
\hline$b d^{\prime}$ & $l$ & $(\bar{b}, \bar{d})$ & 3 \\
\hline$b c^{\prime}$ & $H_{u}+H_{d}$ & $(\bar{b}, \bar{c})+(b, c)$ & 1 \\
\hline$c d$ & $E_{R}$ & $(\bar{c}, d)$ & 3 \\
\hline$c d^{\prime}$ & $N_{R}$ & $(c, d)$ & 3 \\
\hline
\end{tabular}

Table 1: Spectrum for the square quiver.

The perturbatively realized couplings are

$$
\begin{gathered}
<q_{L(1,0,0)}^{I} H_{u(-1,-1,0)} u_{R(0,1,0)}^{J}>\quad<Q_{L(-1,0,0)} H_{d(1,1,0)} d_{R(0,-1,0)}^{I}> \\
<l_{(-1,0,-1)}^{I} H_{d(1,1,0)} E_{R(0,-1,1)}^{J}>.
\end{gathered}
$$

Note that for generic entries in the corresponding mass matrices at this level at least the $u, d$ and $s$ quarks remain massless. There are also no Dirac neutrino masses at this level. On the other hand stringy instantons could give rise to the couplings

$$
\begin{gathered}
<Q_{L(-1,0,0)} H_{u(-1,-1,0)} u_{R(0,1,0)}^{I}>\quad<q_{L(1,0,0)}^{I} H_{d(1,1,0)} d_{R(0,-1,0)}^{J}> \\
<l_{(-1,0,-1)}^{I} H_{u(-1,-1,0)} N_{R(0,1,1)}^{J}>
\end{gathered}
$$

all of which violate the $U(1)_{b}$ symmetry and hence are perturbatively absent. Here the capital letter $I, J$ denote the family index and the subscript indicates the charge under the global $U(1)_{b}, U(1)_{c}$ and $U(1)_{d}$. All three forbidden couplings can be generated non-perturbatively by three different rigid $O(1)$ instantons $E 2_{1}$, $E 2_{2}$ and $E 2_{3}$. In order to have the right charged zero mode structure they have to satisfy

$$
\begin{array}{rlccc}
Q_{L} H_{u} u_{R}^{I}: & \bullet I_{E 2_{1} b}=-1 & I_{E 2_{1} a}=I_{E 2_{1} c}=I_{E 2_{1} d}=0 & \\
& \bullet I_{E 2_{1} b}=-1 & I_{E 2_{1}^{\prime} a}=I_{E 2_{1}^{\prime} c}=I_{E 2_{1}^{\prime} d}=0 & I_{E 2_{1}^{\prime} c}^{N=2}=1 \\
q_{L}^{I} H_{d} d_{R}^{J}: & \bullet I_{E 2_{2} b}=1 & I_{E 2_{2} a}=I_{E 2_{2} c}=I_{E 2_{2} d}=0 & \\
& \bullet & I_{E 2_{2}^{\prime} b}=1 & I_{E 2_{2}^{\prime} a}=I_{E 2_{2}^{\prime} c}=I_{E 2_{2}^{\prime} d}=0 & I_{E 2_{2}^{\prime} c}^{N=2}=1 \\
l^{I} H_{u} N_{R}^{J}: & \bullet I_{E 2_{3} b}=-1 & I_{E 2_{3} a}=I_{E 2_{3} c}=I_{E 2_{3} d}=0 & \\
& \bullet I_{E 2_{3}^{\prime} b}=-1 & I_{E 2_{3}^{\prime} a}=I_{E 2_{3}^{\prime} c}=I_{E 2_{3}^{\prime} d}=0 & I_{E 2_{3}^{\prime} d}^{N=2}=1 \\
& \bullet I_{E 2_{3}^{\prime \prime} b}=-1 & I_{E 2_{3}^{\prime \prime} a}=I_{E 2_{3}^{\prime \prime} c}=I_{E 2_{3}^{\prime \prime} d}=0 & I_{E 2_{3}^{\prime \prime} c}^{N=2}=1 \\
& \bullet I_{E 2_{3}^{\prime \prime \prime} b}=-1 & I_{E 2_{3}^{\prime \prime \prime} a}=I_{E 2_{3}^{\prime \prime \prime} c}=I_{E 2_{3}^{\prime \prime \prime} d}=0 & I_{E 2_{3}^{\prime \prime \prime} c}^{N=2}=I_{E 2_{3}^{\prime \prime \prime} d}^{N=2}=1 .
\end{array}
$$


We begin by discussing the leptonic Yukawa coupling $l^{I} H_{d} N_{R}^{J}$ giving Dirac masses to neutrinos, which can be generated via the four different instantons listed above. Figures 1 a - 1 d display for all four instantons the disk diagrams necessary to soak up all the charged zero modes. The non-perturbative generation of the Yukawa couplings $l^{I} H_{d} N_{r}^{J}$ provides an intriguing explanation for the smallness of the Dirac Neutrino masses [50]. For concreteness let us perform the computation of the non-perturbative contribution arising from an instanton $E 2_{3}$, exhibiting no vector-like charged zero modes. The measure of the path-integral contains all instanton zero modes, the four bosonic and two fermionic universal zero modes, $x^{\mu}$ and $\theta^{\alpha}$, as well as the two charged fermionic zero modes $\lambda_{b}$

$$
\int d^{4} x d^{2} \theta d^{2} \lambda_{b} e^{-S_{E 2_{3}}^{c l} \epsilon_{i j}} \epsilon_{k l}<\lambda_{b}^{i} l^{j} N_{R} H_{u}^{k} \lambda_{b}^{l}>e^{Z^{\prime}}
$$

Here $i, j, k$ and $l$ denote the gauge indices of the $S U(2), e^{-S_{E 2_{3}}^{c l}}$ is the suppression factor of the instanton $E 2_{3}$ and $e^{Z^{\prime}}$ is the regularized one loop amplitude which can be interpreted as one-loop Pfaffian. To avoid an overload in notation we omit the family index 8 . Performing the integral over the charged zero modes results into

$$
\int d^{4} x d^{2} \theta Y_{l H_{u} N_{R}} \epsilon_{i l} l^{i} H_{u}^{l} N_{R}
$$

Here $Y_{l H_{u} N_{R}}$ contains, apart from the classical suppression factor and $e^{Z^{\prime}}$, also the contribution arising from the disk amplitude $\left\langle\lambda_{b} l N_{R} H_{u} \lambda_{b}\right\rangle$, which depends via world-sheet instantons on the open string moduli. To get the desired small masses for the Neutrinos the instanton suppression factor should be in the range of $10^{-13}$ to $10^{-11}$. An analogous analysis can be performed for the other three instantons with the difference that the charged zero modes get soaked up by more than one disk diagram. Note, that for the two instantons $E 2_{3}^{\prime}$ and $E 2_{3}^{\prime \prime \prime}$ the disk diagrams do not contain both matter fields $l^{I}$ and $N_{R}^{J}$, simultaneously. Thus the induced $3 \times 3$ Yukawa coupling matrix factorizes

$$
Y_{l H_{d} N_{r}}^{I J}=Y^{I} Y^{J}
$$

and in the absence of any other instanton the Yukawa coupling $l^{I} H_{d} N_{r}^{J}$ gets generated only for one family. Later we will see, that the factorization of instanton induced Yukawa coupling potentially gives a natural explanation for hierarchies within the class of non-perturbative Yukawa couplings.

Let us turn to the quark Yukawa couplings $Q_{L} H_{u} u_{R}^{I}$ and $q_{L}^{I} H_{d} d_{R}^{J}$. The charged zero modes of $E 2_{1}$ get soaked up by one disk diagram depicted in figure 2a inducing the coupling $Q_{L} H_{u} u_{R}^{I}$. For $E 2_{1}^{\prime}$, exhibiting two additional vectorlike zero modes $\lambda_{c}$ and $\bar{\lambda}_{c}$, the instanton zero modes get saturated via two disk

\footnotetext{
${ }^{8}$ The reader should keep in mind that the instanton induces a $3 \times 3$ Yukawa coupling matrix.
} 
a)

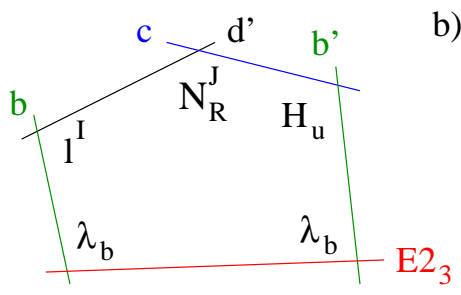

b)

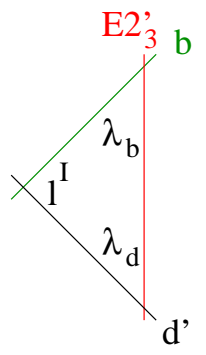

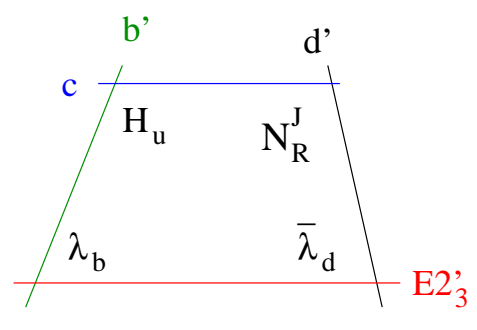

c)

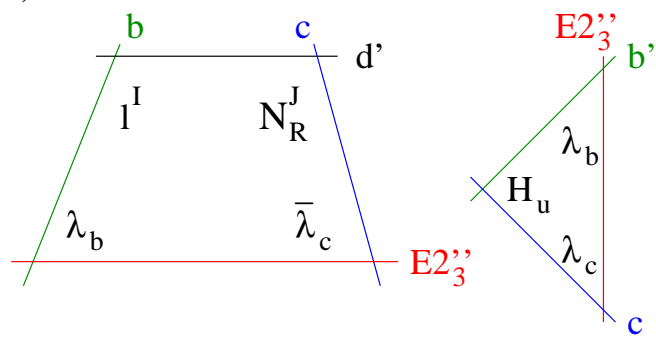

d)

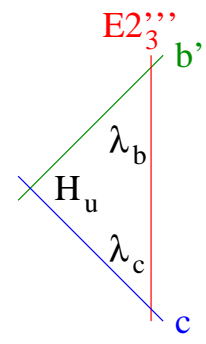

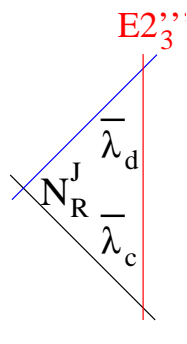

Figure 1: Instanton induced of the Yukawa coupling $l^{I} H_{u} N_{R}^{J}$ for the square quiver.

a)

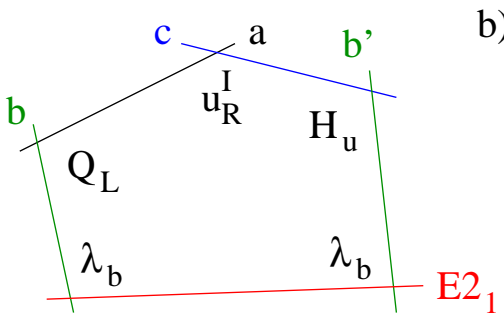

b)

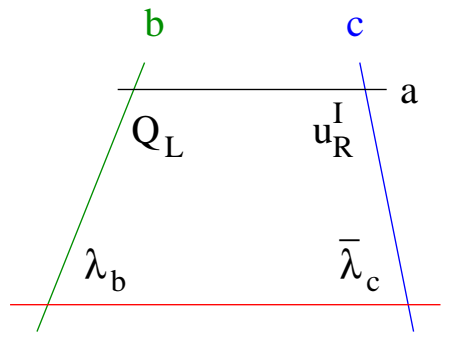

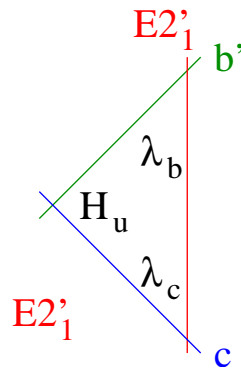

Figure 2: Instanton induced Yukawa coupling $Q_{L} H_{u} u_{R}$ for the square quiver.

diagrams (see figure 2b). Analogously, the other missing quark Yukawa coupling, $q_{L}^{I} H_{d} d_{R}^{I}$ is generated by $E 2_{2}$ or $E 2_{2}^{\prime}$ respectively. In case both instantons $E 2_{i}$ and $E 2_{i}^{\prime}$ are present, the one with the smaller suppression factor $e^{S_{E}^{c l} 2}$ gives the dominant contribution.

Let us now discuss the hierarchies of the quark Yukawa couplings. The fact that only one D-quark gets perturbatively a mass suggests to identify $Q_{L}$ with the left-handed quarks of the third generation. So $q_{L}^{I}$ 's should correspond to the two lightest generations. Then, the suppression factor $e^{-S_{E 2_{1}}^{c l}}\left(e^{-S_{E 2_{1}^{\prime}}^{c l}}\right)$ gives a natural explanation for the smallness of the Yukawa couplings $q_{L}^{I} H_{d} d_{R}$ for the two lightest D-quarks compared to the heaviest. However, due to the same reasoning one would expect a smaller Yukawa coupling $Q_{L} H_{u} u_{R}^{I}$ for the heaviest family. Thus, in contradiction to observations, a smaller mass for the top-quark, 
than for the up- and charm-quark gets generated (or a very large mixing for the third generation is obtained). To be more concrete the perturbatively generated Yukawa coupling matrices take the form

$$
Y_{u_{L}^{I} H_{u} u_{R}^{J}}^{P}=\left(\begin{array}{ccc}
A_{11}^{u} & A_{12}^{u} & A_{13}^{u} \\
A_{21}^{u} & A_{22}^{u} & A_{23}^{u} \\
0 & 0 & 0
\end{array}\right) \quad Y_{d_{L}^{I} H_{d} d_{R}^{J}}^{P}=\left(\begin{array}{ccc}
0 & 0 & 0 \\
0 & 0 & 0 \\
A_{31}^{d} & A_{32}^{d} & A_{33}^{d}
\end{array}\right)
$$

while the non-perturbatively are given by

$$
Y_{u_{L}^{I} H_{u} u_{R}^{J}}^{N P}=\left(\begin{array}{ccc}
0 & 0 & 0 \\
0 & 0 & 0 \\
B_{31}^{u} & B_{32}^{u} & B_{33}^{u}
\end{array}\right) \quad Y_{d_{L}^{I} H_{d} d_{R}^{J}}^{N P}=\left(\begin{array}{ccc}
B_{11}^{d} & B_{12}^{d} & B_{13}^{d} \\
B_{21}^{d} & B_{22}^{d} & B_{23}^{d} \\
0 & 0 & 0
\end{array}\right) .
$$

The total $3 \times 3$ Yukawa coupling matrices are given by the sum of the respective perturbative and non-perturbative part. Generically, due to the instanton suppression factor we expect $A_{i j} \gg B_{k l}$ for arbitrary $i, j, k$ and $l$. Determining the eigenvalues and eigenstates of this matrix reveals a large mixing between the first and third generation which is not observed in nature. Large world-sheet instanton suppressions for specific perturbative realized couplings might relax the expectation $A_{i j} \gg B_{k l}$ and surmount the encountered problem of a too large family mixing. Nevertheless to achieve agreement with experimental observations a lot of fine-tuning is required.

\subsection{Triangle quiver}

Let us turn the triangle quiver with exactly one Higgs pair, where we overcome the issue, of a large fine-tuning to match experimental observations, encountered in the previous setup. Consider the intersection numbers [72]

$$
\begin{array}{rrrrrl}
I_{a b}=1 & I_{a b^{\prime}}=2 & I_{a c}=-3 & I_{a c^{\prime}}=-3 & I_{b d}=-1 \\
I_{b d^{\prime}}=2 & I_{b c}=-1 & I_{b c^{\prime}}=-1 & I_{c d}=3 & I_{c d^{\prime}}=-3,
\end{array}
$$

which give rise to the spectrum displayed in table 2, Tentatively we can asign $Q_{L}$ to the left-handed quarks of the lightest family, while the $q_{l}$ 's would be the left-handed quarks of the other two families. The $L$ is the lepton-doublet of the heaviest family, and the $l$ denotes the $S U(2)$ doublet of the two lightest generations. Note that global consistency requires the existence of additional exotics, charged under the $S U(2)$. The hypercharge is given by the combination

$$
U(1)_{Y}=\frac{1}{3} U(1)_{a}-U(1)_{c}-U(1)_{d} .
$$

Assuming, that all other extra $U(1)$ 's become massive via couplings to RR-fields, the gauge symmetry in four-dimensional spacetime is

$$
S U(3)_{a} \times S U(2)_{b} \times U(1)_{Y} .
$$




\begin{tabular}{|c|c|c|c|}
\hline sector & matter fields & transformation & multiplicities \\
\hline \hline$a b$ & $Q_{L}$ & $(a, \bar{b})$ & 1 \\
\hline$a b^{\prime}$ & $q_{L}$ & $(a, b)$ & 2 \\
\hline$a c$ & $u_{R}$ & $(\bar{a}, c)$ & 3 \\
\hline$a c^{\prime}$ & $d_{R}$ & $(\bar{a}, \bar{c})$ & 3 \\
\hline$b d$ & $L$ & $(\bar{b}, d)$ & 1 \\
\hline$b d^{\prime}$ & $l$ & $(b, d)$ & 2 \\
\hline$b c$ & $H_{d}$ & $(\bar{b}, c)$ & 1 \\
\hline$b c^{\prime}$ & $H_{u}$ & $(\bar{b}, \bar{c})$ & 1 \\
\hline$c d$ & $N_{R}$ & $(c, \bar{d})$ & 3 \\
\hline$c d^{\prime}$ & $E_{R}$ & $(\bar{c}, \bar{d})$ & 3 \\
\hline
\end{tabular}

Table 2: Spectrum for the triangle quiver.

The perturbative Yukawa couplings are

$$
\begin{aligned}
& <q_{L(1,0,0)} H_{u(-1,-1,0)} u_{R(0,1,0)}>\quad<q_{L(1,0,0)} H_{d(-1,1,0)} d_{R(0,-1,0)}> \\
& <l_{(1,0,1)} H_{d(-1,1,0)} E_{R(0,-1,-1)}>\quad<l_{(1,0,1)} H_{u(-1,-1,0)} N_{R(0,1,-1)}>,
\end{aligned}
$$

where the subscripts denote as before the charge under the global $U(1)_{b}, U(1)_{c}$ and $U(1)_{d}$. Note that all fermion mass matrices have then the general structure

$$
m_{U, D, L, N} \simeq\left(\begin{array}{ccc}
A_{11} & A_{12} & A_{13} \\
A_{21} & A_{22} & A_{23} \\
0 & 0 & 0
\end{array}\right)
$$

at the perturbative level. The couplings which are perturbatively forbidden (since they violate $\left.U(1)_{b}\right)$ are

$$
\begin{array}{ll}
<Q_{L(-1,0,0)} H_{u(-1,-1,0)} u_{R(0,1,0)}> & <Q_{L_{(-1,0,0)}} H_{d(-1,1,0)} d_{R(0,-1,0)}> \\
<L_{(-1,0,1)} H_{d(-1,1,0)} E_{R(0,-1,-1)}> & <L_{(-1,0,1)} H_{u(-1,-1,0)} N_{R(0,1,-1)}>
\end{array}
$$

and fill the remaining entries in eq.(20).

Such a setup can be locally realized on the $T^{6} / \mathbb{Z}_{2} \times \mathbb{Z}_{2}^{\prime}$ orientifold where the first two tori are untilted, the third one is tilted and the orientifold charges are chosen to be

$$
\eta_{\Omega \mathcal{R}}=1 \quad \eta_{\Omega \mathcal{R} \theta}=1 \quad \eta_{\Omega \mathcal{R} \theta^{\prime}}=-1 \quad \eta_{\Omega \mathcal{R} \theta \theta^{\prime}}=1
$$

satisfying (62). In appendix $\mathrm{A}$ we present a brief review of this orbifold, which includes a brief discussion of rigid cycles, details of the orientifold action as well as the computation of chiral intersection numbers.

The spacetime-filling D6-branes are given by fractional branes, branes that carry charge only under one twisted sector. These fractional branes wrap the 


\begin{tabular}{|c|c|c|c|}
\hline brane & $\left(n_{1}, m_{1}\right)$ & $\left(n_{2}, m_{2}\right)$ & $\left(n_{3}, \tilde{m}_{3}\right)$ \\
\hline \hline$N_{a}=3$ & $(1,0)$ & $(3,1)$ & $(3,-1 / 2)$ \\
\hline$N_{b}=2$ & $(1,1)$ & $(1,0)$ & $(1,-1 / 2)$ \\
\hline$N_{c}=1$ & $(0,1)$ & $(0,-1)$ & $(2,0)$ \\
\hline$N_{d}=1$ & $(1,0)$ & $(3,1)$ & $(3,-1 / 2)$ \\
\hline
\end{tabular}

Table 3: Bulk wrapping numbers

bulk cycles, displayed in table 3 and their complete homology classes are given by

$$
\begin{aligned}
& \pi_{a}^{F}=\frac{1}{2}[(1,0),(3,1),(3,-1 / 2)]+\frac{1}{2}\left(\sum_{i, j \in(2,4) \times(1,4)}\left[\alpha_{i j}^{\theta} \times[(3,-1 / 2)]\right)\right. \\
& \pi_{b}^{F}=\frac{1}{2}[(1,1),(1,0),(1,-1 / 2)]+\frac{1}{2}\left(\sum_{i, j \in(1,4) \times(1,3)}\left[\alpha_{i j}^{\theta \theta^{\prime}} \times[(1,0)]\right)\right. \\
& \pi_{c}^{F}=\frac{1}{2}[(0,1),(0,-1),(2,0)]+\frac{1}{2}\left(\sum_{i, j \in(1,2) \times(3,4)}\left[\alpha_{i j}^{\theta^{\prime}} \times[(0,1)]\right)\right. \\
& \pi_{d}^{F}=\frac{1}{2}[(1,0),(3,1),(3,-1 / 2)]+\frac{1}{2}\left(\sum_{i, j \in(2,4) \times(2,3)}\left[\alpha_{i j}^{\theta} \times(3,-1 / 2)\right]\right) .
\end{aligned}
$$

The configuration gives rise to a $U(3)_{a} \times U(2)_{b} \times U(1)_{c} \times U(1)_{d}$ gauge theory in four-dimensional spacetime, with the spectrum, computed using equation (67) in appendix A, displayed in table 2 .

Global consistency requires the presence of additional branes. For the hypercharge to be realized as a local symmetry the combination

$$
U_{Y}(1)=\frac{1}{3} U(1)_{a}-U(1)_{c}-U(1)_{d}+\sum_{x} c_{x} U(1)_{x}
$$

has to remain massless. The $U(1)_{x}$ denote the anomalous $U(1)$ 's arising from the additional branes, required for tadpole cancellation. Again for simplicity we assume that this is the only massless combination, all other $U(1)$ 's become massive and survive only as global symmetry. With the choice

$$
U_{1}=\frac{u}{2} \quad U_{2}=\frac{u}{2} \quad U_{3}=u
$$

for the complex structure moduli all D6-branes are aligned to the orientifold plane, thus $\mathcal{N}=1$ supersymmetry in $4 \mathrm{D}$ is ensured. 
In this local D6-brane configuration the couplings, listed in (19), are perturbatively realized, while the Yukawa couplings (21) violate global $U(1)$ selection rules, thus are perturbatively absent. In order to match experimental observation we expect them to be generated via non-perturbative effects.

Before investigating the details of this local realization, let us analyze the non-perturbative effects for a generic triangle quiver, realizing the intersection pattern (16). There are potentially two different types of instanton, $E 2_{1}$ and $E 2_{2}$, both generating all the missing couplings in (21). Their intersection pattern with the matter $D 6$-branes has to satisfy

$$
\begin{aligned}
& I_{E 2_{1} b}=-1 \quad I_{E 2_{1} a}=I_{E 2_{1} c}=I_{E 2_{1} d}=0 \\
& I_{E 2_{2} b}=-1 \quad I_{E 2_{2} a}=I_{E 2_{2} c}=I_{E 2_{2} d}=0 \quad I_{E 2_{1} c}^{\mathcal{N}=2}=1 .
\end{aligned}
$$

In addition there are two classes of instantons $E 2_{3}$ and $E 2_{4}$ with the intersection pattern

$$
\begin{array}{lll}
I_{E 2_{3} b}=-1 & I_{E 2_{3} a}=I_{E 2_{3} c}=I_{E 2_{3} d}=0 & I_{E 2_{3} d}^{\mathcal{N}=2}=1 \\
I_{E 2_{4} b}=-1 & I_{E 2_{4} a}=I_{E 2_{4} c}=I_{E 2_{4} d}=0 & I_{E 2_{4} c}^{\mathcal{N}=2}=I_{E 2_{4} d}^{\mathcal{N}=2}=1
\end{array}
$$

which generate only the lepton Yukawa coupling $L H_{d} E_{R}$ and $L H_{u} N_{R}$.

a)

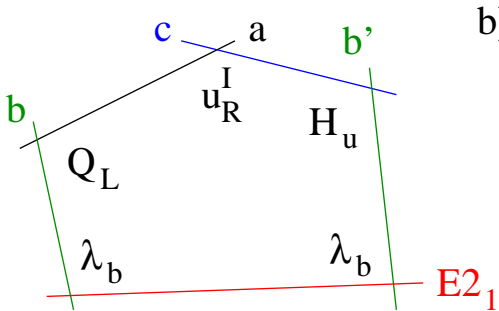

b)

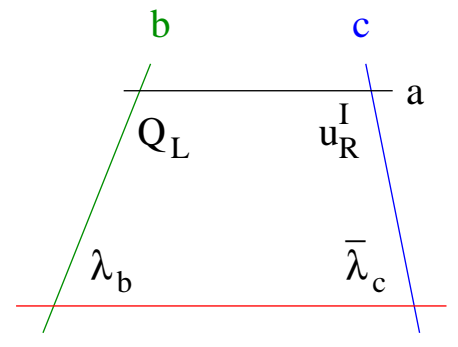

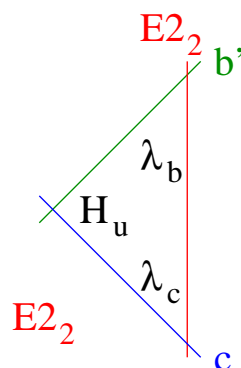

Figure 3: Instanton induced Yukawa coupling $Q_{L} H_{u} u_{R}^{I}$ for the triangle quiver.

Let us now analyze the generation of the U-quark Yukawa coupling $Q_{L} H_{u} u_{R}^{I}$ in more detail. An analogous discussion applies to the coupling $Q_{L} H_{d} d_{R}^{I}$. For the instanton $E 2_{1}$ satisfying (26) the two charged zero modes $\lambda_{b}$ get soaked up by one disk diagram displayed in figure 3 a. The path integral takes the form

$$
\int d^{4} x d^{2} \theta d^{2} \lambda_{b} e^{-S_{E 2}^{c l}} \epsilon_{i j} \epsilon_{k l}<\lambda_{b}^{i} Q_{L}^{j} u_{R}^{I} H_{u}^{k} \lambda_{b}^{l}>e^{Z^{\prime}}
$$

which, after performing the integral over the charged fermionic zero modes, results into

$$
\int d^{4} x d^{2} \theta Y_{Q_{L} H_{u} u_{R}^{I}} \epsilon_{i l} Q_{L}^{i} H_{u}^{l} u_{R}^{I}
$$


The indices $i, j, k$ and $l$ label again the flavor charge, the capital letter denotes the family and $Y_{Q_{L} H_{u} u_{R}^{I}}$ is the final effective Yukawa coupling, including the

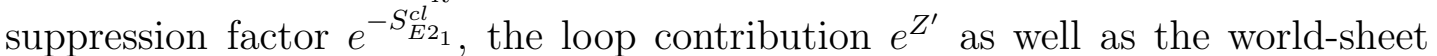
contribution arising from the disk diagram $<\lambda_{b} Q_{L} u_{R}^{I} H_{u} \lambda_{b}>$.

For the instanton $E 2_{2}$, with an additional pair of vector-like charged zero modes, one performs a similar computation with the difference that the four charged zero modes get saturated via two disk diagrams (see figure 3b)

$$
\int d^{4} x d^{2} \theta d^{2} \lambda_{b} d \bar{\lambda}_{c} d \lambda_{c} e^{-S_{E 2_{2}}^{c l} \epsilon_{i j}}<\lambda_{b}^{i} Q_{L}^{j} u_{R}^{I} \bar{\lambda}_{c}>\epsilon_{k l}<\lambda_{b}^{k} H_{u}^{l} \lambda_{c}>e^{Z^{\prime}},
$$

which gives the superpotential contribution

$$
\int d^{4} x d^{2} \theta Y_{Q_{L} H_{u} u_{R}^{I}}^{\prime} \epsilon_{i l} Q_{L}^{i} H_{u}^{l} u_{R}^{I} .
$$

In case, both types of instantons are present, the one, which wraps the smaller cycle in the internal manifold, thus exhibiting a smaller suppression factor in the path integral, gives the dominant contribution to the Yukawa couplings. To match experimental data the suppression factor of the dominant contribution is expected to be of the order $10^{-2}-10^{-5}$.

An analogous analysis applies to the lepton Yukawa couplings $l^{I} H_{d} E_{R}{ }^{J}$ and $l^{I} H_{u} N_{R}{ }^{J}$, with the difference that there are potentially two additional classes of instantons $E 2_{3}$ and $E 2_{4}$ which can induce these couplings. Again the dominant contribution arises from the instanton, which wraps the smallest cycle in the internal manifold. Note, that in opposite to the previous setup here the Dirac Neutrino masses are expected to be of the same order as the masses of charged lepton. One possibility to obtain small masses for neutrinos is due to the so called see-saw mechanism. For this mechanism to work a large Majorana mass term for the right-handed Neutrinos is required. Such a mass term could be generated via an instanton $E 2_{x}$ with the intersection pattern $[18,20]$

$$
I_{E 2_{x} c}=I_{E 2_{x} d}=2 \quad I_{E 2_{x} a}=I_{E 2_{x} b}=0 .
$$

With a suppression factor of the order $10^{-8}$ to $10^{-5}$ the Majorana mass term $M_{N_{R}}$ for the right-handed Neutrino lies in the range $10^{10} \mathrm{GeV}<M_{N_{R}}<10^{13} \mathrm{GeV}$. Together with the Dirac masses for the Neutrinos, which are of the electroweak scale $(0.01-1) \mathrm{GeV}$ that would give the see-saw Neutrino masses in the desired range $\left(10^{-2}-10^{-1}\right) \mathrm{eV}$.

After the analysis for a generic triangle quiver satisfying the intersection pattern (16), let us turn to our concrete realization and examine if it exhibits instantons, inducing the perturbatively forbidden couplings. Indeed, as we will see momentarily the local realization comprises one representant for each of the first two classes, $E 2_{1}$ and $E 2_{2}$. 
An instanton wrapping the cycle

$$
\begin{aligned}
\pi_{E 2_{1}} & =\frac{1}{4}[(1,0)(0,1)(0,-1)]+\frac{1}{4}\left(\sum_{i, j \in(1,3) \times(3,4)}\left[\alpha_{i j}^{\theta} \times(0,-1)\right]\right) \\
& +\frac{1}{4}\left(\sum_{i, j \in(3,4) \times(1,2)}\left[\alpha_{i j}^{\theta^{\prime}} \times(1,0)\right]\right)+\frac{1}{4}\left(\sum_{i, j \in(1,3) \times(1,2)}\left[\alpha_{i j}^{\theta \theta^{\prime}} \times[(0,1)]\right)\right.
\end{aligned}
$$

gives rise to the intersection pattern in (26) . Note, that all $\mathcal{N}=2$ modes between the instanton and the D6-branes are massive (see also appendix $\mathrm{B}$ ). Moreover, the cycle $\pi_{E 2_{1}}$ is rigid and invariant under the orientifold action $\Omega \mathcal{R}$, thus the instanton contains only the four bosonic zero modes $x^{\mu}$ and the two fermionic $\theta^{\alpha}$. Therefore, the instanton $E 2_{1}$ indeed gives rise to all the perturbatively missing couplings in (21). Its suppression factor is

$$
e^{-S_{E 2_{1}}^{c l}}=e^{-\frac{2 \pi}{l_{s}^{3} g_{s}} \operatorname{Vol}_{E 2_{1}}}=e^{-\frac{2 \pi}{\alpha_{a}} \frac{\mathrm{Vol}_{E 2_{1}}}{\mathrm{Vol}_{D 6_{a}}}},
$$

where the ratio $\frac{\operatorname{Vol}_{E 2_{1}}}{\operatorname{Vol}_{D 6_{a}}}$ is given by

$$
\frac{\mathrm{Vol}_{E 2_{1}}}{\operatorname{Vol}_{D 6_{a}}}=\frac{1}{2}\left(\prod_{I}\left[\frac{\left(n_{E 2_{1}}^{I}\right)^{2}+\left(\widetilde{m}_{E 2_{1}}^{I}\right)^{2} U_{I}^{2}}{\left(n_{a}^{I}\right)^{2}+\left(\widetilde{m}_{a}^{I}\right)^{2} U_{I}^{2}}\right]\right)^{1 / 2}
$$

The factor $\frac{1}{2}$ is due to the fact that the $D 6$-brane wraps a fractional cycle while the instanton a rigid one (see the different prefactors in equation (23) and (35)). If we assume $\alpha_{a}=1 / 24$ at string scale and the complex structure moduli $U_{3}=u$ stabilized in the range $(1-1.5)$ we get the desired suppression factor

$$
e^{-S_{E 2_{1}}^{c l}} \sim 10^{-2}-10^{-5}
$$

There is also a representant $E 2_{2}$ for the second class of instantons. It wraps the cycle

$$
\begin{aligned}
\pi_{E 2_{2}} & =\frac{1}{4}[(1,0)(0,1)(0,-1)]+\frac{1}{4}\left(\sum_{i, j \in(1,3) \times(1,2)}\left[\alpha_{i j}^{\theta} \times(0,-1)\right]\right) \\
& +\frac{1}{4}\left(\sum_{i, j \in(1,2) \times(1,2)}\left[\alpha_{i j}^{\theta^{\prime}} \times(1,0)\right]\right)+\frac{1}{4}\left(\sum_{i, j \in(1,3) \times(1,2)}\left[\alpha_{i j}^{\theta \theta^{\prime}} \times[(0,1)]\right)\right.
\end{aligned}
$$

and gives rise to the same chiral intersection pattern as the instanton $E 2_{1}$, but there also vectorlike pairs of charged zero modes in the $\mathcal{N}=2$ sector between instanton $E 2_{2}$ and the $D 6$-branes. We relegate the detailed analysis of the $\mathcal{N}=2$ 
instanton zero mode sector to the appendix $\mathrm{B}$. There we show that indeed exactly one vectorlike pair $\lambda_{c}, \bar{\lambda}_{c}$ arises at the intersection $E 2_{2}$ and $c$. Thus, such an instanton induces as well as $E 2_{1}$ all the missing couplings in (21). Since the $E 2_{2}$ wraps the same bulk-cycle as $E 2_{1}$ their suppression factors are equal

$$
e^{-S_{E 2_{1}}^{c l}}=e^{-S_{E 2_{2}}^{c l}} \sim 10^{-2}-10^{-5}
$$

and their contributions to the Yukawa couplings are expected of the same order.

The non-perturbative contributions depend via the disk diagrams on the world-sheet instantons, thus on the area, that the diagrams enclose. Therefore the dominant contribution to the Yukawa couplings arises from that instanton, whose disk-diagrams encloses the smallest area.

This concrete realization does not exhibit an instanton, which could give rise to large Majorana mass term for the right-handed Neutrino $N_{R}$. Thus at this level the Neutrino masses are of the same order as the masses for their doublet partner. Note also, that in this explicit local setup the perturbative contribution to fermion mass matrices in eq.(20) factorizes, due to the fact that the non-trivial intersections for the family index carrying matter fields occur in different two tori [13]. Thus, the perturbative matrix has rank one and one fermion per generation remains massless even after instanton corrections are taken into account.

a)

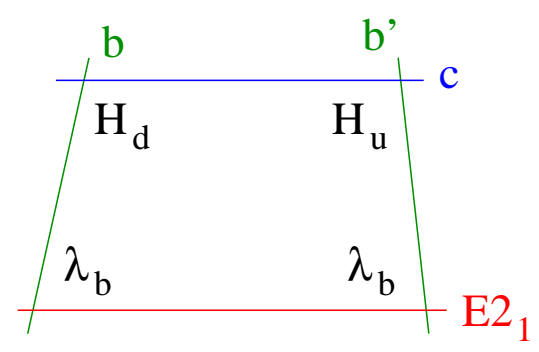

b)

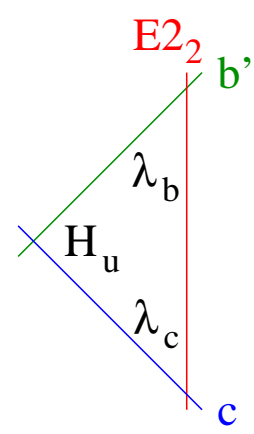

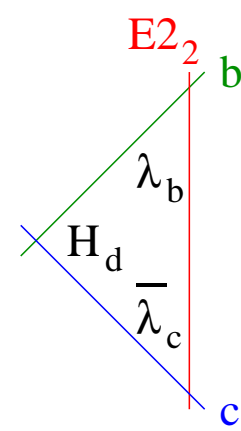

Figure 4: Non perturbative generation of the $\mu$-term for the triangle quiver.

Finally let us draw attention to a phenomenological drawback of the simplest triangle quiver. The Yukawa coupling inducing instantons, $E 2_{1}$ and $E 2_{2}$, generate also a $\mu$-term for the Higgs pair (see figure 4)

$$
<H_{u(-1,-1)} H_{d(-1,1)}>,
$$

which is far too large. To match the Yukawa couplings with experimental observations, we expect the instanton suppression factor $e^{-S_{E 2}^{c l}}$ to be in the range of $10^{-2}$ to $10^{-5}$. But then the same instanton would induce a $\mu$-term of the order 
$\left(10^{13}-10^{16}\right) \mathrm{GeV}$ rather than $\left(10^{2}-10^{3}\right) \mathrm{GeV}$. In the next section we examine a setup, where we allow for a second Higgs pair and show that one can generate all the missing Yukawa couplings without inducing a too large $\mu$-term for the Higgs pair.

\subsection{Triangle quiver with doubled Higgs sector}

A way to avoid the previously encountered problem of generating a too large $\mu$ term is to allow for a doubled Higgs sector. The hope would be, that the Yukawa coupling generating instanton induces a $\mu$-term matrix which factorizes. Thus it only generates for one Higgs pair a $\mu$-term of the order $10^{13}-10^{15} \mathrm{GeV}$, while the other one, the physical one, remains massless. The $\mu$-term for the physical MSSM-Higgs pair is generated by a different instanton, which wraps a larger cycle, leading to a larger suppression factor and possibly to a $\mu$-term of the order $\left(10^{2}-10^{3}\right) \mathrm{GeV}$. If the supermassive unphysical Higgs doublets do not acquire a vev they can be integrated out and do not affect the low energy effective action.

In the following we will discuss the realization of such a setup. The intersection numbers are equivalent to the previous case up to the $b c$ and $b c^{\prime}$-sector, which are doubled, respectively,

$$
\begin{array}{lrrrrl}
I_{a b}=1 & I_{a b^{\prime}}=2 & I_{a c}=-3 & I_{a c^{\prime}}=-3 & I_{b d}=-1 \\
I_{b d^{\prime}}=2 & I_{b c}=-2 & I_{b c^{\prime}}=-2 & I_{c d}=3 & I_{c d^{\prime}}=-3 .
\end{array}
$$

Note, that this implies the same number of 2 and $\overline{2}$, fulfilling the constraint, arising from tadpole cancellation. Table 4 displays the origin, the transformation behavior and the multiplicities of the matter fields. Again, we require that apart

\begin{tabular}{|c|c|c|c|}
\hline sector & matter fields & transformation & multiplicities \\
\hline \hline$a b$ & $Q_{L}$ & $(a, \bar{b})$ & 1 \\
\hline$a b^{\prime}$ & $q_{L}$ & $(a, b)$ & 2 \\
\hline$a c$ & $u_{R}$ & $(\bar{a}, c)$ & 3 \\
\hline$a c^{\prime}$ & $d_{R}$ & $(\bar{a}, \bar{c})$ & 3 \\
\hline$b d$ & $L$ & $(\bar{b}, d)$ & 1 \\
\hline$b d^{\prime}$ & $l$ & $(b, d)$ & 2 \\
\hline$b c$ & $H_{d}$ & $(\bar{b}, c)$ & 2 \\
\hline$b c^{\prime}$ & $H_{u}$ & $(\bar{b}, \bar{c})$ & 2 \\
\hline$c d$ & $N_{R}$ & $(c, \bar{d})$ & 3 \\
\hline$c d^{\prime}$ & $E_{R}$ & $(\bar{c}, \bar{d})$ & 3 \\
\hline
\end{tabular}

Table 4: Spectrum

from the hypercharge

$$
U(1)_{Y}=\frac{1}{3} U(1)_{a}-U(1)_{c}-U(1)_{d}
$$


all other U(1)'s become massive and survive only as global symmetries. The perturbatively allowed couplings are

$$
\begin{aligned}
& <q_{L(1,0,0)} H_{u(-1,-1,0)}^{I} u_{R(0,1,0)}>\quad<q_{L(1,0,0)} H_{d(-1,1,0)}^{I} d_{R(0,-1,0)}> \\
& <l_{(1,0,1)} H_{d(-1,1,0)}^{I} E_{R(0,-1,-1)}>\quad<l_{(0,1,0)} H_{u(0,-1,-1)}^{I} N_{R(0,1,-1)}>.
\end{aligned}
$$

The $U(1)$ selection rules violating and thus forbidden couplings are given by

$$
\begin{aligned}
& <Q_{L_{(-1,0,0)} H_{u(-1,-1,0)}^{I} u_{R(0,1,0)}>} \quad<Q_{L_{(-1,0,0)}} H_{d(-1,1,0)}^{I} d_{R(0,-1,0)}> \\
& <L_{(-1,0,1)} H_{d(-1,1,0)}^{I} E_{R(0,-1,-1)}>\quad<L_{(-1,0,1)} H_{u(-1,-1,0)}^{I} N_{R(0,1,-1)}>,
\end{aligned}
$$

where $I$ denotes the family index of the Higgs doublets and we suppress the matter field family index.

For the background $T^{6} / \mathbb{Z}_{2} \times \mathbb{Z}_{2}^{\prime}$ orientifold, with the orientifold charges chosen as before (see equation (22) ) and the last two tori tilted, a local realization of the intersection numbers (42) is given by four fractional D6-branes wrapping the bulk-cycles displayed in table 5 .

\begin{tabular}{|c|c|c|c|}
\hline brane & $\left(n_{1}, m_{1}\right)$ & $\left(n_{2}, m_{2}\right)$ & $\left(n_{3}, \tilde{m}_{3}\right)$ \\
\hline \hline$N_{a}=3$ & $(1,0)$ & $(3,1 / 2)$ & $(3,-1 / 2)$ \\
\hline$N_{b}=2$ & $(1,1)$ & $(2,0)$ & $(1,-1 / 2)$ \\
\hline$N_{c}=1$ & $(0,1)$ & $(0,-1)$ & $(2,0)$ \\
\hline$N_{d}=1$ & $(1,0)$ & $(3,1 / 2)$ & $(3,-1 / 2)$ \\
\hline
\end{tabular}

Table 5: Bulk wrapping numbers

Their complete homology classes are given by

$$
\begin{aligned}
& \pi_{a}^{F}=\frac{1}{2}[(1,0),(3,1 / 2),(3,-1 / 2)]+\frac{1}{2}\left(\sum_{i, j \in(2,4) \times(1,3)}\left[\alpha_{i j}^{\theta \theta^{\prime}} \times(3,1 / 2)\right]\right) \\
& \pi_{b}^{F}=\frac{1}{2}[(1,1),(2,0),(1,-1 / 2)]+\frac{1}{2}\left(\sum_{i, j \in(1,4) \times(3,4)}\left[\alpha_{i j}^{\theta} \times(1,-1 / 2)\right]\right) \\
& \pi_{c}^{F}=\frac{1}{2}[(0,1),(0,-1),(2,0)] \quad+\frac{1}{2}\left(\sum_{i, j \in(1,2) \times(3,4)}\left[\alpha_{i j}^{\theta^{\prime}} \times(0,1)\right]\right) \\
& \pi_{d}^{F}=\frac{1}{2}[(1,0),(3,1 / 2),(3,-1 / 2)]+\frac{1}{2}\left(\sum_{i, j \in(2,4) \times(2,4)}\left[\alpha_{i j}^{\theta \theta^{\prime}} \times(3,1 / 2)\right]\right)
\end{aligned}
$$

and again we assume that the hypercharge

$$
U_{Y}(1)=\frac{1}{3} U(1)_{a}-U(1)_{c}-U(1)_{d}+\sum_{x} c_{x} U(1)_{x}
$$


remains massless after including the additional branes required for global consistency. To ensure supersymmetry we choose the complex structure moduli to be

$$
U_{1}=\frac{u}{2} \quad U_{2}=u \quad U_{3}=u
$$

The perturbatively forbidden couplings in (45) can be generated by two classes of instantons, either by an instanton with only two charged zero modes

$$
I_{E 2_{2} b}=-1 \quad I_{E 2_{2} a}=I_{E 2_{2} c}=I_{E 2_{2} d}=0 .
$$

or by an instanton with four charged zero modes

$$
I_{E 2_{2} b}=-1 \quad I_{E 2_{2} a}=I_{E 2_{2} c}=I_{E 2_{2} d}=0 \quad I_{E 2_{2} c}^{\mathcal{N}=2}=1
$$

As for the triangle quiver with just one Higgs pair there are two additional classes of instantons

$$
\begin{array}{lll}
I_{E 2_{3} b}=-1 & I_{E 2_{3} a}=I_{E 2_{3} c}=I_{E 2_{3} d}=0 & I_{E 2_{3} d}^{\mathcal{N}=2}=1 \\
I_{E 2_{4} b}=-1 & I_{E 2_{4} a}=I_{E 2_{3} c}=I_{E 2_{4} d}=0 & I_{E 2_{4} c}^{\mathcal{N}=2}=I_{E 2_{4} d}^{\mathcal{N}=2}=1
\end{array}
$$

which generate only the lepton Yukawa couplings $L H_{d}^{I} E_{R}$ and $L H_{u}^{I} N_{R}$, but not any of the quark Yukawa couplings.

The analysis of the lepton and quark Yukawa couplings is very similar and all results apply for this setup as well. As previously, $E 2_{1}$ and $E 2_{2}$ give rise to a $\mu$-term there

$$
\mu_{I J} H_{d}^{I} H_{u}^{J}
$$

for the Higgs fields. In opposite to triangle quiver discussed in section 3.2 , the $\mu$-term is a $2 \times 2$ matrix. In case the instanton, inducing the missing couplings (45), gives rise to a factorizable $\mu$-term matrix, only one Higgs pair receives mass of order $\left(10^{13}-10^{16}\right) \mathrm{GeV}$, while the other one, which we identify with the physical MSSM-Higgs pair, remains massless. Then potentially another instanton, wrapping a larger cycle in the internal manifold could generate additional non-perturbative corrections to the Yukawa-couplings and the $\mu$-term matrix. Due to the higher suppression factor these corrections are negligible for all couplings, apart for the mass term for the physical Higgs pair, for which it gives the dominant contribution. In case the suppression factor arising from the second instanton is of the order $e^{-S_{E 2}^{c l}} \sim 10^{-16}-10^{-15}$ the $\mu$-term is in the desired range $\left(10^{2}-10^{3}\right) \mathrm{GeV}$.

We now discuss under which circumstances the $2 \times 2$ matrix $\mu_{I J}$ factorizes, thus leaving only one Higgs pair massless. For an instanton $E 2_{1}$ satisfying (49) the charged zero modes $\bar{\lambda}_{b}$ get soaked by just one disk diagram (see figure $5 \mathrm{a}$ )

$$
B^{I J}=<\lambda_{b} H_{d}^{I} H_{u}^{J} \lambda_{b}>
$$


Here, there is no reason to expect a factorization of the matrix $\mu_{I J} \sim B^{I J}$, thus both Higgs multiplets get a mass of the order $e^{-S_{E 2_{1}}^{c l}} M_{s}$. For the other class of instantons, $E 2_{2}$, satisfying (50), the four charged zero modes get soaked up by two disk diagrams (see figure $5 \mathrm{~b}$ )

$$
A_{d}^{I}=<\lambda_{b} H_{d}^{I} \bar{\lambda}_{c}>\quad \text { and } \quad A_{u}^{J}=<\lambda_{b} H_{u}^{J} \lambda_{c}>.
$$

Clearly, for this class of instantons the matrix $\mu_{I J} \sim A_{d}^{I} A_{u}^{J}$ factorizes, thus only one of the Higgs pairs acquires a mass of the order $e^{-S_{E 2_{2}}^{c l}} M_{s}$. The other, the physical, Higgs pair remains massless. Thus, in a setup satisfying (42), which exhibits both classes of instantons, Yukawa couplings of the right order can be generated without inducing a too large $\mu$-term for the physical Higgs pair. The instanton $E 2_{2}$ of the second type gives rise to the correct texture of the Yukawa couplings, while the suppression factor, $e^{-S_{E 2_{1}}^{c l}}$, of the instanton $E 2_{1}$ is of the right order to induce a $\mu$-term for the physical Higgs pair.

The linear combination remaining light depends on the values of the worldsheet amplitudes $A_{d}^{I}, A_{u}^{J}$. Note that now, unlike the simplest triangle setup, the perturbative contribution to the fermion mass matrices eq.(20) has generically rank two, since the massless physical field is a linear combination of two fields with different worldsheet couplings.

a)

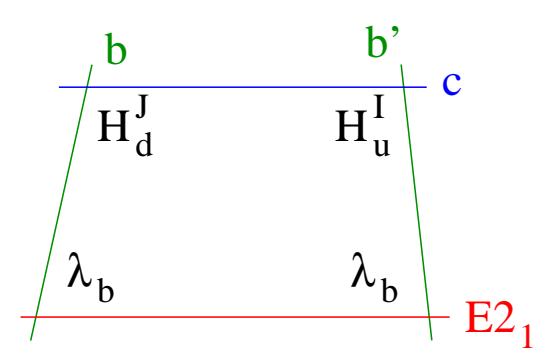

b)

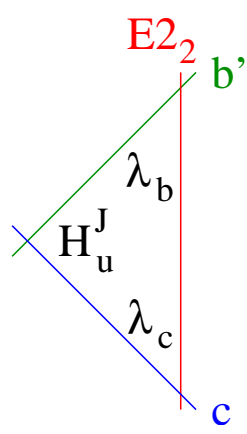

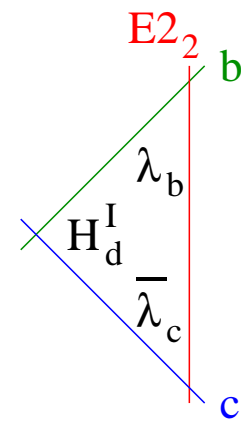

Figure 5: Non-perturbative generation of $\mu$-term matrix $\mu^{I J}$.

For the local realization (46) only a representant of the second type, satisfying (50), is present. It gives rise to all perturbatively missing couplings listed in (45). The instanton $E 2$ wraps the orientifold invariant cycle

$$
\begin{aligned}
\pi_{E 2} & =\frac{1}{4}[(1,0)(0,1)(0,-1)]+\frac{1}{4}\left(\sum_{i, j \in(1,3) \times(1,2)}\left[\alpha_{i j}^{\theta} \times(0,-1)\right]\right) \\
& +\frac{1}{4}\left(\sum_{i, j \in(1,2) \times(1,2)}\left[\alpha_{i j}^{\theta^{\prime}} \times(1,0)\right]\right)+\frac{1}{4}\left(\sum_{i, j \in(1,3) \times(1,2)}\left[\alpha_{i j}^{\theta \theta^{\prime}} \times[(0,1)]\right)\right.
\end{aligned}
$$


and has the intersection pattern (50). For the analysis of the $\mathcal{N}=2$ sector we refer the reader to the appendix B. To obtain realistic Yukawa couplings the suppression factor should be in the range $10^{-2}-10^{-5}$ which can be achieved, via (36) and (37), if the complex structure moduli $U_{3}=u$ is stabilized in the range $(0.7-1)$. Here we assume again that the $S U(3)$ gauge coupling is approximately $\alpha_{a}=1 / 24$ at the string scale.

As discussed above the instanton $E 2_{2}$ also induces a large $2 \times 2 \mu$-term matrix for the Higgs pairs, which factorizes. Thus, only one Higgs pair gets supermassive, while the other one remains massless. While in principle the $\mu$-term for the physical Higgs pair can be generated by a different instanton, wrapping a larger cycle in the internal manifold, this local example does not exhibit such an instanton.

Analogous to the previous setup the smallness of the Neutrino masses could be explained by the see-saw mechanism. The instanton inducing Majorana mass term for the right-handed Neutrino has to satisfy (34) and is expected to have a suppression factor of the order $10^{-8}-10^{-5}$. For the local realization (46) such an instanton is absent.

Summarizing we see that the triangle quiver with a doubled Higgs sector can give rise to Yukawa couplings of the right texture and at the same time also exhibit a $\mu$-term of the desired order. After integrating out the superheavy Higgs pair the low effective action resembles the one of the MSSM. Furthermore, in case the setup also exhibits an instanton with the right suppression factor, satisfying (34), the see-saw mechanism can be realized, thus the origin of the smallness of the Neutrino masses could be explained.

\section{Conclusion}

In this work we have analyzed MSSM-like quivers with respect to their Yukawa couplings. Specifically we have focused on three different configurations, each consisting of four different stacks of D-branes, the square-quiver, the trianglequiver and the triangle-quiver with a doubled Higgs sector. We have shown that in principle in all three configurations all perturbatively absent, but desired couplings could be generated via stringy instantons.

Furthermore, we investigated the phenomenological implications of these nonperturbative effects. The square-quiver, discussed in section 3.1, does not allow for a perturbative realization of the Dirac Neutrino masses, thus the exponentially suppressed non-perturbative generation for this Yukawa coupling gives an intriguing explanation for the smallness of the Neutrino masses. However, the square-quiver generically gives rise to a large mixing between the first and third generation for the quark Yukawa couplings, which requires a large fine-tuning to overcome.

For the triangle-quiver we show that such fine-tuning is not necessary to 
match experimental observations. All perturbatively absent couplings can potentially generated by a single instanton. Clearly, in this case the neat mechanism for small Neutrino masses, encountered for the square quiver does not apply. However, the setup, realizing the triangle quiver, might in principle exhibit an instanton inducing a large Majorana mass term for the right-handed Neutrinos, thus realizing the see-saw mechanism. Nevertheless for the local realizations of both triangle quivers no such instanton is found. The simplest triangle quiver has a generic potential drawback. The very same instanton generating all missing Yukawa couplings, induces also a $\mu$-term for the Higgs pair, which is far too large.

As we discuss in section 3.3, if we allow for a doubled Higgs sector then this phenomenological drawback might be surmounted. While the analysis for the Yukawa-couplings is very similar to the triangle quiver with just one Higgs pair, the $\mu$-term arising from the Yukawa generating instanton is a $2 \times 2$ matrix. In case the instanton induced $\mu$-term matrix factorizes only one of the Higgs pairs becomes supermassive while the other one, which we identify with physical MSSM Higgs pair, remains massless. A second instanton with a larger suppression factor could then induce the $\mu$ term for the MSSM Higgs pair. In section 3.3 we show that a setup, realizing the triangle-quiver with a doubled Higgs sector, potentially exhibits a class of instantons, generating all the missing Yukawa couplings and giving rise a $\mu$-term matrix, which factorizes.

For both triangle quivers, discussed in section 3.2 and 3.3 , we provide local realizations within the $T^{6} / \mathbb{Z}_{2} \times \mathbb{Z}_{2}^{\prime}$ orientifold with torsion, which gives rise to rigid cycles. We show that they exhibit instantons which generate the missing Yukawa couplings with the right hierarchies. For the triangle quiver with a doubled Higgs sector the Yukawa coupling inducing instanton gives rise to a $\mu$-term matrix which factorizes. Thus for this local setup we can realize all Yukawa coupling with the right hierarchies without encountering any phenomenological drawbacks. However, this local setup does not exhibit an instanton which could generate a $\mu$-term of the order $\left(10^{2}-10^{3}\right) \mathrm{GeV}$ for the MSSM Higgs pair.

We leave it for future work to extend the analysis to other quivers, such as quivers based on three stacks of D-branes and quivers leading to GUT-like spectrum. Moreover, it would be nice to find a global realization of the discussed quivers, which satisfies the severe constraints on the instanton zero mode structure. This seems to be more promising in the T-dual Type I framework. Finally, it would be interesting to perform a detailed analysis of the MSSM-like Gepner orientifold constructions, provided in [76,77], with respect to their Yukawa couplings. 


\section{Acknowledgments}

We thank M. Ambroso, T. Brelidze, M. Cvetič, I. García-Etxebarria, C. Kokorelis, F. Marchesano, A. Uranga and T. Weigand for useful discussions. This work has been supported by the European Commission under RTN European Programs MRTN-CT-2004-503369, MRTN-CT-2004-005105, by the CICYT (Spain) under project FPA2006-01105, the Comunidad de Madrid under project HEPHACOS P-ESP-00346 and the Ingenio 2010 CONSOLIDER program CPAN. 


\section{A $\quad T^{6} / \mathbb{Z}_{2} \times \mathbb{Z}_{2}^{\prime}$ Orientifold}

This appendix is dedicated to brief review of the $T^{6} / \mathbb{Z}_{2} \times \mathbb{Z}_{2}^{\prime}$ orientifold with Hodge numbers $\left(h_{11}, h_{12}\right)=(3,51)$. We adopt the notation of [75], to which we refer the reader for further details. The orbifold group consists of two generators $\theta$ and $\theta^{\prime}$ acting as reflection in the first two and last two tori, respectively, while there combination $\theta \theta^{\prime}$ amounts into a reflection in the first and third torus.

As usual there are the bulk cycles

$$
\Pi_{a}^{B}=4 \bigotimes_{I=1}^{3}\left(n_{a}^{I}\left[a^{I}\right]+\widetilde{m}_{a}^{I}\left[b^{I}\right]\right)
$$

defined in terms of the fundamental one-cycles $\left[a^{I}\right],\left[b^{I}\right]$ of the $I$-th $T^{2}$ and the corresponding wrapping numbers $n_{a}^{I}$ and $\widetilde{m}_{a}^{I}=m_{a}^{I}+\beta^{I} n_{a}^{I}$. Here $\beta^{I}$ takes the value 0 and $1 / 2$ for rectangular and tilted tori, respectively.

In addition this backgrounds gives rise to another class of cycles, the so called $g$-twisted cycles. All three actions, $\theta, \theta^{\prime}$ and $\theta \theta^{\prime}$ exhibit 16 fixed points which after blowing up give rise to two-cycles with the topology of $\mathbb{P}_{1}$. In combination with the fundamental one-cycle invariant under the respective action they construct the $g$-twisted cycles

$$
\Pi_{i j}^{g}=\left[\alpha_{i j}^{g} \times\left(n^{I_{g}}, \widetilde{m}^{I_{g}}\right)\right] .
$$

Here $i, j \in\{1,2,3,4\} \times\{1,2,3,4\}$ labels one of the 16 blown-up fixed points of the orbifold element $g=\theta, \theta^{\prime}, \theta \theta^{\prime} \in \mathbb{Z}_{2} \times \mathbb{Z}_{2}^{\prime}$ and $I_{g}$ denotes the $g$-invariant one-cycle with $I_{g}=3,1,2$ for $g=\theta, \theta^{\prime}, \theta \theta^{\prime}$.

Rigid cycles are charged under all three sectors $\theta, \theta^{\prime}$ and $\theta \theta^{\prime}$ and take the form

$$
\Pi^{F}=\frac{1}{4} \Pi^{B}+\frac{1}{4}\left(\sum_{i, j \in S_{\theta}} \epsilon_{i j}^{\theta} \Pi_{i j}^{\theta}\right)+\frac{1}{4}\left(\sum_{j, k \in S_{\theta^{\prime}}} \epsilon_{j k}^{\theta^{\prime}} \Pi_{j k}^{\theta^{\prime}}\right)+\frac{1}{4}\left(\sum_{i, k \in S_{\theta \theta^{\prime}}} \epsilon_{i k}^{\theta \theta^{\prime}} \Pi_{i k}^{\theta \theta^{\prime}}\right),
$$

where $S_{g}$ is the set of fixed points in the $g$-twisted sector. The $\epsilon_{i j}^{g}= \pm 1$ correspond to the two different orientation the brane can wrap the blown up $\mathbb{P}_{1}$ and are subject to various consistency conditions [75].

The orientifold action $\Omega \mathcal{R}$ for the bulk cycles takes the usual form

$$
\Omega \mathcal{R}:\left[\left(n_{1}, \widetilde{m}_{1}\right)\left(n_{2}, \widetilde{m}_{2}\right)\left(n_{3}, \widetilde{m}_{3}\right)\right] \rightarrow\left[\left(n_{1},-\widetilde{m}_{1}\right)\left(n_{2},-\widetilde{m}_{2}\right)\left(n_{3},-\widetilde{m}_{3}\right)\right] .
$$

For the $g$-twisted cycle $\Omega \mathcal{R}$ acts

$$
\Omega \mathcal{R}:\left[\alpha_{i j}^{g} \times\left(n^{I_{g}}, \widetilde{m}^{I_{g}}\right)\right] \rightarrow \eta_{\Omega \mathcal{R}} \eta_{\Omega \mathcal{R} g}\left[\alpha_{\mathcal{R}(i) \mathcal{R}(j)}^{g} \times\left(-n^{I_{g}}, \widetilde{m}^{I_{g}}\right)\right],
$$

where $\eta_{\Omega \mathcal{R} g}= \pm 1$ denote the orientifold charges of the different sectors and are subject to the constraint

$$
\eta_{\Omega \mathcal{R}} \eta_{\Omega \mathcal{R} \theta} \eta_{\Omega \mathcal{R} \theta^{\prime}} \eta_{\Omega \mathcal{R} \theta \theta^{\prime}}=-1
$$




\begin{tabular}{|c|c|}
\hline Representation & Multiplicity \\
\hline$\Xi_{a}$ & $\frac{1}{2}\left(\pi_{a} \circ \pi_{a}^{\prime}+\pi_{a} \circ \pi_{\mathrm{O} 6}\right)$ \\
$\square_{a}$ & $\frac{1}{2}\left(\pi_{a} \circ \pi_{a}^{\prime}-\pi_{a} \circ \pi_{\mathrm{O} 6}\right)$ \\
$(a, \bar{b})$ & $\pi_{a} \circ \pi_{b}$ \\
$(a, b)$ & $\pi_{a} \circ \pi_{b}^{\prime}$ \\
\hline
\end{tabular}

Table 6: Chiral spectrum for intersecting D6-branes.

The reflection $\mathcal{R}$ leaves all fixed points of an untilted two-torus invariant and acts on the fixed points in a tilted two-torus as

$$
\mathcal{R}(1)=1, \quad \mathcal{R}(2)=2, \quad \mathcal{R}(3)=4, \quad \mathcal{R}(4)=3 .
$$

With the orientifold action (60) the fixed point locus is

$$
\begin{aligned}
\pi_{O 6}= & 2^{3} \eta_{\Omega \mathcal{R}}[(1,0)(1,0)(1,0)]+2^{3-2 \beta_{1}-2 \beta_{2}} \eta_{\Omega \mathcal{R} \theta}[(0,1)(0,-1)(1,0)] \\
& +2^{3-2 \beta_{2}-2 \beta_{3}} \eta_{\Omega \mathcal{R} \theta^{\prime}}[(1,0)(0,1)(0,-1)]+2^{3-2 \beta_{1}-2 \beta_{3}} \eta_{\Omega \mathcal{R} \theta \theta^{\prime}}[(0,-1)(1,0)(0,1)]
\end{aligned}
$$

Generically, the chiral spectrum is given by the topological intersection numbers displayed in table [6 ${ }^{9}$. Given two branes $a$ and $b$ in the $T^{6} / \mathbb{Z}_{2} \times \mathbb{Z}_{2}^{\prime}$ orbifold background, the topological intersection numbers for the bulk part is

$$
\pi_{a}^{B} \circ \pi_{b}^{B}=4 \prod_{i=1}^{3}\left(n_{a}^{i} \widetilde{m}_{b}^{i}-n_{b}^{i} \widetilde{m}_{a}^{i}\right)
$$

and for the twisted sector takes the form

$$
\left[\alpha_{i j}^{g} \times\left(n_{a}^{I_{g}}, \widetilde{m}_{a}^{I_{g}}\right)\right] \circ\left[\alpha_{k l}^{h} \times\left(n_{b}^{I_{h}}, \widetilde{m}_{b}^{I_{h}}\right)\right]=4 \delta_{i k} \delta_{j l} \delta^{g h}\left(n_{a}^{I_{g}} \widetilde{m}_{b}^{I_{h}}-n_{b}^{I_{h}} \widetilde{m}_{a}^{I_{g}}\right) .
$$

In both local realizations presented in section 3.2 and 3.3 the matter $D 6$-branes wrap fractional cycles, cycles which are charged only under one twisted sector $g$

$$
\pi^{F}=\frac{1}{2} \pi^{B}+\frac{1}{2}\left(\sum_{i, j \in S_{g}} \epsilon_{i j}^{g}\left[\alpha_{i j}^{g} \times\left(n_{a}^{I_{g}}, \widetilde{m}_{a}^{I_{g}}\right)\right]\right) .
$$

This class of cycles are only rigid in two tori and can move freely in the torus invariant under the action $g$. Then, for two fractional branes charged under a different twisted sector the intersection number is simply given by

$$
\pi_{a}^{F} \circ \pi_{b}^{F}=\prod_{i=1}^{3}\left(n_{a}^{i} \widetilde{m}_{b}^{i}-n_{b}^{i} \widetilde{m}_{a}^{i}\right)
$$

\footnotetext{
${ }^{9}$ Note that we choose the convention that positive intersection number $\pi_{a} \circ \pi_{b}$ corresponds to matter transforming as $(a, \bar{b})$.
} 
To achieve global consistency the RR charges of the branes and orientifolds have to be cancelled 10 . That translates into conditions on the bulk and twisted sector of the branes. Since we are only interested in local constructions we ignore them and refer the interested reader to [75].

Finally, to ensure supersymmetry all branes have to be aligned to the orientifold plane. This amounts into two constraints each brane has to satisfy. Expressed in terms of the wrapping numbers they are

$$
\widetilde{m}^{1} \widetilde{m}^{2} \widetilde{m}^{3}-\sum_{I \neq J \neq K} \frac{n^{I} n^{J} \widetilde{m}^{K}}{U^{I} U^{J}}=0
$$

and

$$
n^{1} n^{2} n^{3}-\sum_{I \neq J \neq K} \widetilde{m}^{I} \widetilde{m}^{J} n^{K} U^{I} U^{J}>0
$$

where $U^{I}$ denotes the complex structure modulus $U^{I}=R_{Y}^{I} / R_{X}^{I}$ of the $I-t h$ torus with radii $R_{X}^{I}$ and $R_{Y}^{I}$.

\section{B $\mathcal{N}=2$ instanton zero mode sector}

This appendix deals with the detailed analysis of the $\mathcal{N}=2$ instantonic zero modes arising in the examples presented in section 3.2 and 3.3. We follow closely the analysis performed in chapter 5 of [75].
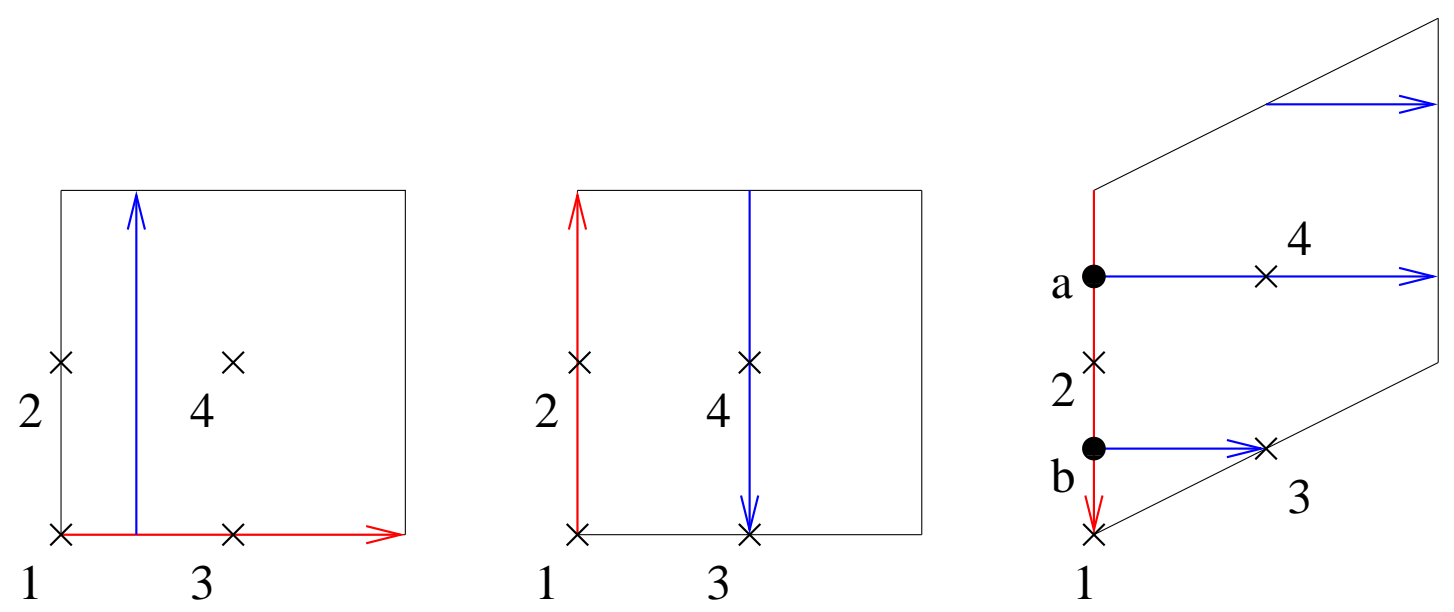

Figure 6: Brane $c$ (blue) and the instanton $E 2_{1}$ (red).

Consider the four stack model giving rise to just one Higgs pair discussed in section 3.2. The D6-branes configuration exhibits two different instantons $E 2_{1}$

\footnotetext{
${ }^{10}$ Moreover, there are K-theory constraints which have to be satisfied.
} 
and $E 2_{2}$ satisfying the two intersection pattern

$$
\begin{array}{lll}
I_{E 2_{1} b}=-1 & I_{E 2_{1} a}=I_{E 2_{1} c}=I_{E 2_{1} d}=0 \\
I_{E 2_{2} b}=-1 & I_{E 2_{2} a}=I_{E 2_{2} c}=I_{E 2_{2} d}=0 & I_{E 2_{1} c}^{\mathcal{N}=2}=1 .
\end{array}
$$

For $E 2_{1}$ there are no massless vector-like states between the $D 6$-branes and the instanton, while for $E 2_{2}$ there is exactly one massless vectorlike state arising at the intersection of the instanton $E 2_{2}$ and the $D 6$-brane $c$.

Let us start by analyzing the $\mathcal{N}=2$ sector for the instanton $E 2_{1}$, whereas we focus on the $E 2-c$ sector. An analogous discussion applies for all the other $E 2_{1}-D 6$ sectors. Figure 6 depicts the intersection between the instanton $E 2_{1}$ and D-brane $c$. Note, that the two cycles $D 6_{c}$ and $E 2_{1}$ wrap (see equations (23) and (35) ), are parallel in the second torus, but go through different fixed points. Thus both cycles are separated in the second torus, the $\mathcal{N}=2$ modes are massive and one does not observe any massless vectorlike states in $E 2_{1}-D 6_{c}$ sector. A similar analysis reveals that there are no vectorlike states in any of the other $E 2_{1}-D 6$ sectors.

For the instanton $E 2_{2}$ similar arguments explain the absence of massless vectorlike states for all $E 2_{2}-D 6$ sectors apart from the $E 2_{2}-c$ sector. In opposite to $E 2_{1}, E 2_{2}$ and $c$ are not separated in the second torus, but both $E 2_{2}$ and $c$ pass through the same fixed points (see figure 7). Thus the $\mathcal{N}=2$ sector does not get massive and one potentially observes massless vectorlike states.
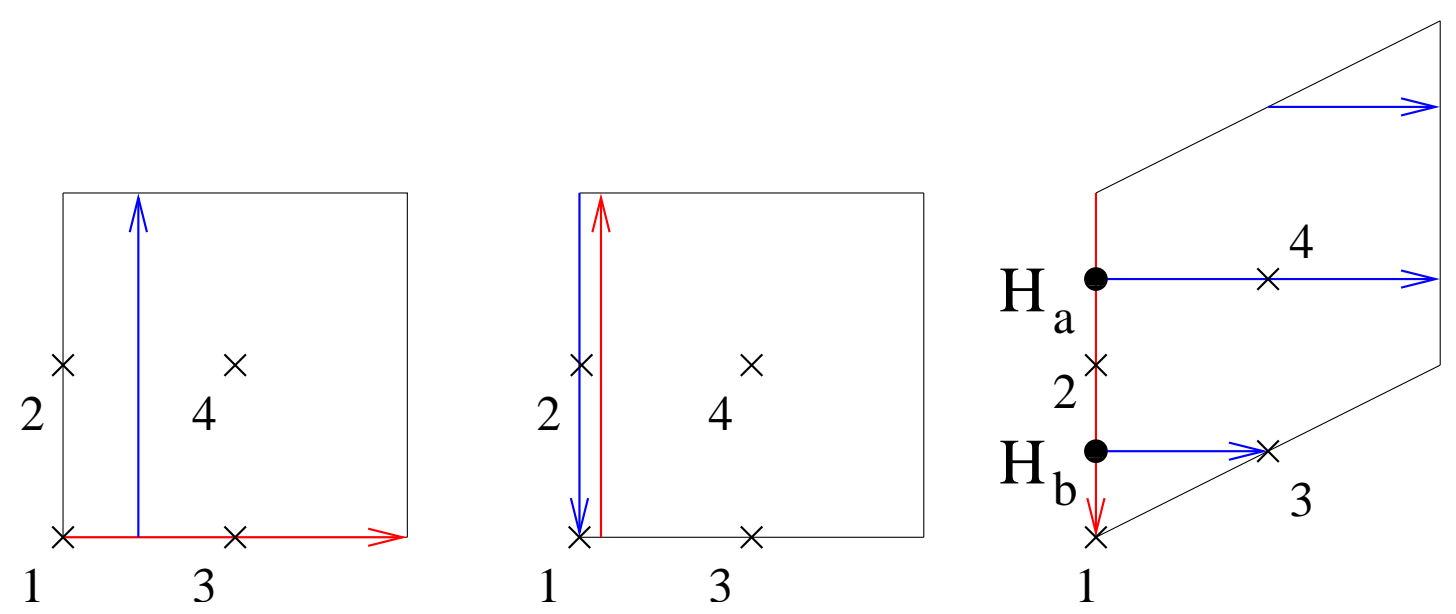

Figure 7: Brane $c$ (blue) and the instanton $E 2_{2}$ (red).

Subsequently we analyze how many of them are present. The bulk sector implies the presence of 2 hypermultiplets in the ambient space11, which we denote

\footnotetext{
${ }^{11}$ The number of hypermultiplets is given by the number of intersections in the first and third torus $I_{E 2_{2} c^{\text {bulk }}}^{\mathcal{N}=2}=\left(n_{E 2_{2}}^{1} m_{c}^{1}-n_{c}^{1} m_{E 2_{2}}^{1}\right)\left(n_{E 2_{2}}^{3} m_{c}^{3}-n_{c}^{3} m_{E 2_{2}}^{3}\right)$.
} 
by $H_{a}$ and $H_{b}$ (see figure 7). Including the orbifold action, which acts as

$$
\begin{aligned}
\theta: & & \left(H_{a}, H_{b}\right) \rightarrow\left(H_{a}, H_{b}\right) \\
\theta^{\prime}: & & \left(H_{a}, H_{b}\right) \rightarrow\left(H_{b}, H_{a}\right) \\
\theta \theta^{\prime}: & & \left(H_{a}, H_{b}\right) \rightarrow\left(H_{b}, H_{a}\right)
\end{aligned}
$$

we see that only the combination $H_{a}+H_{b}$ survives. Thus there is indeed exactly one charged vectorlike pair of zero modes, $\lambda_{c}$ and $\bar{\lambda}_{c}$, in the $E 2_{2}-c$ sector.
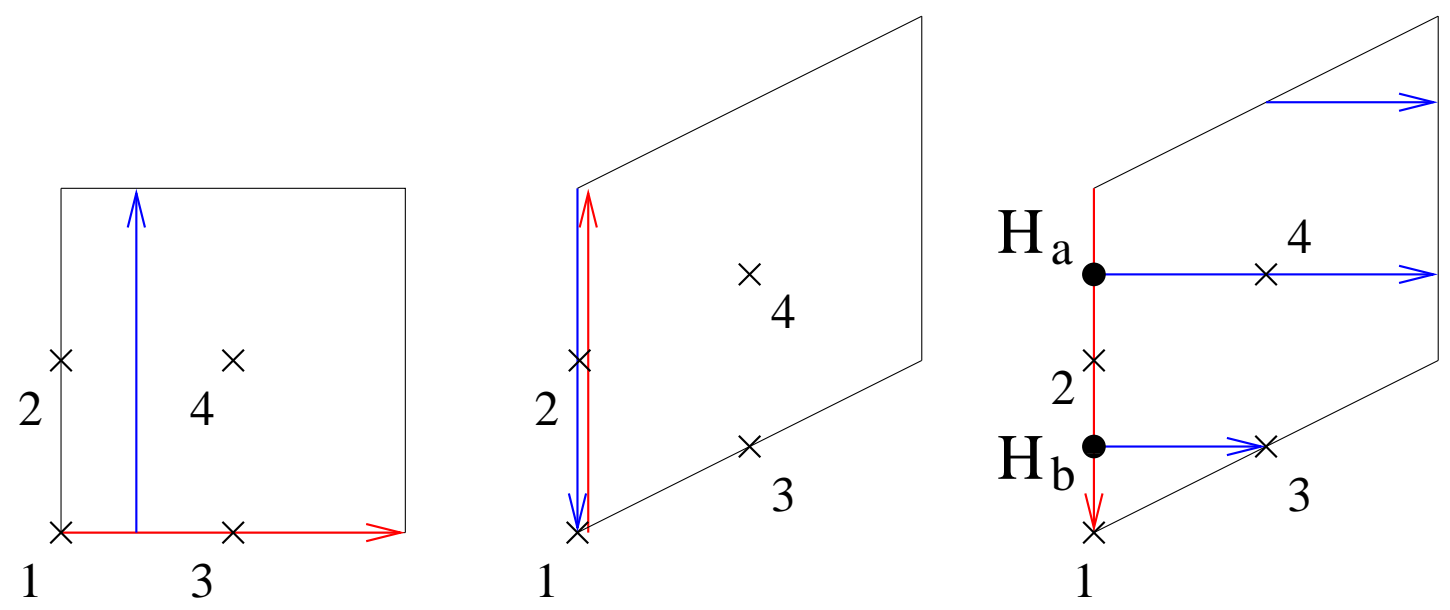

Figure 8: Brane $c$ (blue) and the instanton $E 2$ (red).

Let us turn to the example presented in section 3.3. This D6-brane configuration comprises an instanton with the intersection pattern

$$
I_{E 2_{2} b}=-1 \quad I_{E 2_{2} a}=I_{E 2_{2} c}=I_{E 2_{2} d}=0 \quad I_{E 2_{2} c}^{\mathcal{N}=2}=1 .
$$

Analogously to the setup above there are no massless vectorlike states between the instanton $E 2$ and $D 6$-brane $a, b$ and $d$. Figure 8 displays the intersection between the cycles the instanton $E 2$ and the D-brane $c$ wrap. They lie on top of each other in the second torus, giving potentially massless $\mathcal{N}=2$ states in the $E 2-c$ sector. As previously, in the ambient space we have 2 hypermultiplets, which are subject to the orbifold action. A similar analysis as above reveals that only one combination, $H_{a}+H_{b}$ survives this action. Thus as desired we have exactly 2 instanton zero modes charged under the global $U(1)_{c}$, namely $\lambda_{c}$ and $\bar{\lambda}_{c}$. 


\section{References}

[1] R. Blumenhagen, M. Cvetič, P. Langacker, and G. Shiu, "Toward realistic intersecting D-brane models," Ann. Rev. Nucl. Part. Sci. 55 (2005) 71-139, hep-th/0502005.

[2] R. Blumenhagen, B. Körs, D. Lüst, and S. Stieberger, "Four-dimensional String Compactifications with D-Branes, Orientifolds and Fluxes," Phys. Rept. 445 (2007) 1-193, hep-th/0610327.

[3] F. Marchesano, "Progress in D-brane model building," Fortsch. Phys. 55 (2007) 491-518, hep-th/0702094.

[4] R. Blumenhagen, L. Görlich, B. Körs, and D. Lüst, "Noncommutative compactifications of type I strings on tori with magnetic background flux," JHEP 10 (2000) 006, hep-th/0007024.

[5] G. Aldazabal, S. Franco, L. E. Ibáñez, R. Rabadán, and A. M. Uranga, "D = 4 chiral string compactifications from intersecting branes," J. Math. Phys. 42 (2001) 3103-3126, hep-th/0011073.

[6] G. Aldazabal, S. Franco, L. E. Ibáñez, R. Rabadán, and A. M. Uranga, "Intersecting brane worlds," JHEP 02 (2001) 047, hep-ph/0011132.

[7] R. Blumenhagen, B. Körs, D. Lüst, and T. Ott, "The standard model from stable intersecting brane world orbifolds," Nucl. Phys. B616 (2001) 3-33, hep-th/0107138.

[8] M. Cvetič, G. Shiu, and A. M. Uranga, "Three-family supersymmetric standard like models from intersecting brane worlds," Phys. Rev. Lett. 87 (2001) 201801, hep-th/0107143.

[9] M. Cvetič, G. Shiu, and A. M. Uranga, "Chiral four-dimensional N = 1 supersymmetric type IIA orientifolds from intersecting D6-branes," Nucl. Phys. B615 (2001) 3-32, hep-th/0107166.

[10] S. A. Abel and A. W. Owen, "Interactions in intersecting brane models," Nucl. Phys. B663 (2003) 197-214, hep-th/0303124.

[11] M. Cvetič and I. Papadimitriou, "Conformal field theory couplings for intersecting D-branes on orientifolds," Phys. Rev. D68 (2003) 046001, hep-th/0303083.

[12] D. Lüst, P. Mayr, R. Richter, and S. Stieberger, "Scattering of gauge, matter, and moduli fields from intersecting branes," Nucl. Phys. B696 (2004) 205-250, hep-th/0404134. 
[13] D. Cremades, L. E. Ibáñez, and F. Marchesano, "Yukawa couplings in intersecting D-brane models," JHEP 07 (2003) 038, hep-th/0302105.

[14] M. Cvetič, P. Langacker, and G. Shiu, "A three-family standard-like orientifold model: Yukawa couplings and hierarchy," Nucl. Phys. B642 (2002) 139-156, hep-th/0206115.

[15] C.-M. Chen, T. Li, V. E. Mayes, and D. V. Nanopoulos, "A Realistic World from Intersecting D6-Branes," Phys. Lett. B665 (2008) 267-270, hep-th/0703280.

[16] C.-M. Chen, T. Li, V. E. Mayes, and D. V. Nanopoulos, "Towards realistic supersymmetric spectra and Yukawa textures from intersecti ng branes," Phys. Rev. D77 (2008) 125023, 0711.0396.

[17] C.-M. Chen, T. Li, V. E. Mayes, and D. V. Nanopoulos, "Yukawa Corrections from Four-Point Functions in Intersecting D6-Brane Models," 0807.4216 .

[18] R. Blumenhagen, M. Cvetič, and T. Weigand, "Spacetime instanton corrections in 4D string vacua - the seesaw mechanism for D-brane models," Nucl. Phys. B771 (2007) 113-142, hep-th/0609191.

[19] M. Haack, D. Krefl, D. Lüst, A. Van Proeyen, and M. Zagermann, "Gaugino condensates and D-terms from D7-branes," JHEP 01 (2007) 078, hep-th/0609211.

[20] L. E. Ibáñez and A. M. Uranga, "Neutrino Majorana masses from string theory instanton effects," JHEP 03 (2007) 052, hep-th/0609213.

[21] B. Florea, S. Kachru, J. McGreevy, and N. Saulina, "Stringy instantons and quiver gauge theories," JHEP 05 (2007) 024, hep-th/0610003.

[22] S. A. Abel and M. D. Goodsell, "Realistic Yukawa couplings through instantons in intersecting brane worlds," JHEP 10 (2007) 034, hep-th/0612110.

[23] N. Akerblom, R. Blumenhagen, D. Lüst, E. Plauschinn, and M. Schmidt-Sommerfeld, "Non-perturbative SQCD Superpotentials from String Instantons," JHEP 04 (2007) 076, hep-th/0612132.

[24] M. Bianchi and E. Kiritsis, "Non-perturbative and Flux superpotentials for Type I strings on the Z3 orbifold," Nucl. Phys. B782 (2007) 26-50, hep-th/0702015. 
[25] M. Cvetič, R. Richter, and T. Weigand, "Computation of D-brane instanton induced superpotential couplings - Majorana masses from string theory," Phys. Rev. D76 (2007) 086002, hep-th/0703028.

[26] R. Argurio, M. Bertolini, S. Franco, and S. Kachru, "Metastable vacua and D-branes at the conifold," JHEP 06 (2007) 017, hep-th/0703236.

[27] R. Argurio, M. Bertolini, G. Ferretti, A. Lerda, and C. Petersson, "Stringy Instantons at Orbifold Singularities," JHEP 06 (2007) 067, arXiv:0704.0262 [hep-th].

[28] M. Bianchi, F. Fucito, and J. F. Morales, "D-brane Instantons on the T6/Z3 orientifold," JHEP 07 (2007) 038, arXiv:0704.0784 [hep-th].

[29] L. E. Ibáñez, A. N. Schellekens, and A. M. Uranga, "Instanton Induced Neutrino Majorana Masses in CFT Orientifolds with MSSM-like spectra," JHEP 06 (2007) 011, arXiv:0704.1079 [hep-th].

[30] N. Akerblom, R. Blumenhagen, D. Lüst, and M. Schmidt-Sommerfeld, "Instantons and Holomorphic Couplings in Intersecting D- brane Models," JHEP 08 (2007) 044, arXiv:0705.2366 [hep-th].

[31] S. Antusch, L. E. Ibáñez, and T. Macri, "Neutrino Masses and Mixings from String Theory Instantons," JHEP 09 (2007) 087, arXiv:0706.2132 [hep-ph].

[32] R. Blumenhagen, M. Cvetič, D. Lüst, R. Richter, and T. Weigand, "Non-perturbative Yukawa Couplings from String Instantons," Phys. Rev. Lett. 100 (2008) 061602, 0707.1871.

[33] O. Aharony and S. Kachru, "Stringy Instantons and Cascading Quivers," JHEP 09 (2007) 060, arXiv:0707.3126 [hep-th].

[34] O. Aharony, S. Kachru, and E. Silverstein, "Simple Stringy Dynamical SUSY Breaking," Phys. Rev. D76 (2007) 126009, arXiv:0708.0493 [hep-th].

[35] R. Blumenhagen, M. Cvetič, R. Richter, and T. Weigand, "Lifting D-Instanton Zero Modes by Recombination and Background Fluxes," JHEP 10 (2007) 098, arXiv:0708.0403 [hep-th].

[36] M. Billó et al., "Instantons in N=2 magnetized D-brane worlds," JHEP 10 (2007) 091, arXiv:0708.3806 [hep-th].

[37] M. Billó et al., "Instanton effects in $\mathrm{N}=1$ brane models and the Kahler metric of twisted matter," JHEP 12 (2007) 051, arXiv:0709.0245 [hep-th]. 
[38] M. Aganagic, C. Beem, and S. Kachru, "Geometric Transitions and Dynamical SUSY Breaking," Nucl. Phys. B796 (2008) 1-24, arXiv:0709.4277 [hep-th].

[39] P. G. Camara, E. Dudas, T. Maillard, and G. Pradisi, "String instantons, fluxes and moduli stabilization," Nucl. Phys. B795 (2008) 453-489, arXiv:0710.3080 [hep-th].

[40] M. Cvetič and T. Weigand, "Hierarchies from D-brane instantons in globally defined Calabi-Yau Orientifolds," arXiv:0711.0209 [hep-th].

[41] L. E. Ibáñez and A. M. Uranga, "Instanton Induced Open String Superpotentials and Branes at Singularities," JHEP 02 (2008) 103, arXiv:0711.1316 [hep-th].

[42] I. García-Etxebarria and A. M. Uranga, "Non-perturbative superpotentials across lines of marginal stability," JHEP 01 (2008) 033, arXiv:0711.1430 [hep-th].

[43] C. Petersson, "Superpotentials From Stringy Instantons Without Orientifolds," arXiv:0711.1837 [hep-th].

[44] R. Blumenhagen, S. Moster, and E. Plauschinn, "Moduli Stabilisation versus Chirality for MSSM like Type IIB Orientifolds," JHEP 01 (2008) 058, arXiv:0711.3389 [hep-th].

[45] M. Bianchi and J. F. Morales, "Unoriented D-brane Instantons vs Heterotic worldsheet Instantons," JHEP 02 (2008) 073, arXiv:0712.1895 [hep-th].

[46] R. Blumenhagen and M. Schmidt-Sommerfeld, "Power Towers of String Instantons for N=1 Vacua," JHEP 07 (2008) 027, 0803.1562.

[47] Y. Matsuo, J. Park, C. Ryou, and M. Yamamoto, "D-instanton derivation of multi-fermion F-terms in supersymmetric QCD," JHEP 06 (2008) 051, 0803.0798 .

[48] R. Argurio, G. Ferretti, and C. Petersson, "Instantons and Toric Quiver Gauge Theories," JHEP 07 (2008) 123, 0803.2041.

[49] M. Cvetič, R. Richter, and T. Weigand, "(Non-)BPS bound states and D-brane instantons," JHEP 07 (2008) 012, 0803.2513.

[50] M. Cvetič and P. Langacker, "D-Instanton Generated Dirac Neutrino Masses," 0803.2876.

[51] I. García-Etxebarria, F. Marchesano, and A. M. Uranga, "Non-perturbative F-terms across lines of BPS stability," JHEP 07 (2008) 028, 0805.0713. 
[52] M. Buican and S. Franco, "SUSY breaking mediation by D-brane instantons," 0806.1964.

[53] D. Forcella, I. García-Etxebarria, and A. Uranga, "E3-brane instantons and baryonic operators for D3-branes on toric singularities," 0806.2291.

[54] R. Blumenhagen, S. Moster, and E. Plauschinn, "String GUT Scenarios with Stabilised Moduli," 0806.2667.

[55] P. G. Camara and E. Dudas, "Multi-instanton and string loop corrections in toroidal orbifold models," JHEP 08 (2008) 069, 0806.3102.

[56] M. Billó et al., "Flux interactions on D-branes and instantons," 0807.1666.

[57] M. Cvetič and T. Weigand, "A string theoretic model of gauge mediated supersymmetry breaking," 0807.3953.

[58] M. Billó et al., "Non-perturbative effective interactions from fluxes," 0807.4098 .

[59] J. Kumar, "A Toy Model for Gauge-Mediation in Intersecting Brane Models," 0808.1264.

[60] J. Marsano, N. Saulina, and S. Schäfer-Nameki, "Gauge Mediation in F-Theory GUT Models," 0808.1571.

[61] J. Marsano, N. Saulina, and S. Schäfer-Nameki, "An Instanton Toolbox for F-Theory Model Building," 0808.2450.

[62] A. M. Uranga, "D-brane instantons and the effective field theory of flux compactifications," 0808.2918.

[63] J. J. Heckman and C. Vafa, "F-theory, GUTs, and the Weak Scale," 0809.1098 .

[64] I. García-Etxebarria, "D-brane instantons and matrix models," 0810.1482.

[65] T. Jeliński and J. Pawelczyk, "Multi-Instanton Corrections to Superpotentials in Type II Compactifications," 0810.4369.

[66] N. Akerblom, R. Blumenhagen, D. Lüst, and M. Schmidt-Sommerfeld, "D-brane Instantons in 4D Supersymmetric String Vacua," Fortsch. Phys. 56 (2008) 313-323, 0712.1793.

[67] M. Cvetič, R. Richter, and T. Weigand, "D-brane instanton effects in Type II orientifolds: local and global issues," 0712.2845. 
[68] E. Witten, "Non-Perturbative Superpotentials In String Theory," Nucl. Phys. B474 (1996) 343-360, hep-th/9604030.

[69] O. J. Ganor, "A note on zeroes of superpotentials in F-theory," Nucl. Phys. B499 (1997) 55-66, hep-th/9612077.

[70] M. Billó et al., "Classical gauge instantons from open strings," JHEP 02 (2003) 045, hep-th/0211250.

[71] L. E. Ibáñez, F. Marchesano, and R. Rabadán, "Getting just the standard model at intersecting branes," JHEP 11 (2001) 002, hep-th/0105155.

[72] D. Cremades, L. E. Ibáñez, and F. Marchesano, "Intersecting brane models of particle physics and the Higgs mechanism," JHEP 07 (2002) 022, hep-th/0203160.

[73] D. Cremades, L. E. Ibáñez, and F. Marchesano, "More about the standard model at intersecting branes," hep-ph/0212048.

[74] F. Marchesano and G. Shiu, "Building MSSM flux vacua," JHEP 11 (2004) 041, hep-th/0409132.

[75] R. Blumenhagen, M. Cvetič, F. Marchesano, and G. Shiu, "Chiral D-brane models with frozen open string moduli," JHEP 03 (2005) 050, hep-th/0502095.

[76] T. P. T. Dijkstra, L. R. Huiszoon, and A. N. Schellekens, "Supersymmetric standard model spectra from RCFT orientifolds," Nucl. Phys. B710 (2005) $3-57$, hep-th/0411129.

[77] P. Anastasopoulos, T. P. T. Dijkstra, E. Kiritsis, and A. N. Schellekens, "Orientifolds, hypercharge embeddings and the standard model," Nucl. Phys. B759 (2006) 83-146, hep-th/0605226. 